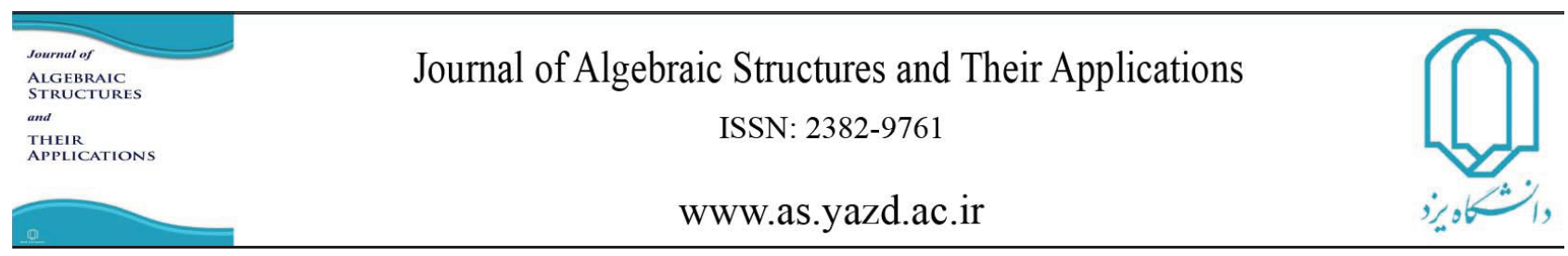

Algebraic Structures and Their Applications Vol. 4 No. 1 ( 2017 ) pp 1-18.

\title{
SMALL GRAPHS WITH EXACTLY TWO NON-NEGATIVE EIGENVALUES
}

\author{
TAJEDIN DERIKVAND AND MOHAMMAD REZA OBOUDI*
}

Communicated by M.A. Iranmanesh

\begin{abstract}
Let $G$ be a graph with eigenvalues $\lambda_{1}(G) \geq \cdots \geq \lambda_{n}(G)$. In this paper we find all simple graphs $G$ such that $G$ has at most twelve vertices and $G$ has exactly two non-negative eigenvalues. In other words we find all graphs $G$ on $n$ vertices such that $n \leq 12$ and $\lambda_{1}(G) \geq 0, \lambda_{2}(G) \geq 0$ and $\lambda_{3}(G)<0$. We obtain that there are exactly 1575 connected graphs $G$ on $n \leq 12$ vertices with $\lambda_{1}(G)>0, \lambda_{2}(G)>0$ and $\lambda_{3}(G)<0$. We find that among these 1575 graphs there are just two integral graphs.
\end{abstract}

\section{Introduction}

Throughout this paper all graphs are simple, that is finite and undirected without loops and multiple edges. Let $G$ be a graph with vertex set $\left\{v_{1}, \ldots, v_{n}\right\}$. The adjacency matrix of $G, A(G)=\left[a_{i j}\right]$, is an $n \times n$ matrix such that $a_{i j}=1$ if $v_{i}$ and $v_{j}$ are adjacent, and $a_{i j}=0$, otherwise. Thus $A(G)$ is a symmetric matrix with zeros on the diagonal and all the eigenvalues of $A(G)$ are real. By the eigenvalues of $G$ we mean those of its adjacency matrix. We denote the eigenvalues of $G$ by $\lambda_{1}(G) \geq \cdots \geq \lambda_{n}(G)$. By the spectrum of $G$ that is denoted by $\operatorname{Spec}(G)$, we mean the multiset of eigenvalues of $G$. The characteristic polynomial of $G$, $\operatorname{det}(\lambda I-A(G))$, is denoted by $P(G, \lambda)$. An integral graph is defined http://dx.doi.org/10.29252/asta.4.1.1

MSC(2010): 05C31; 05C50; 05C75; 05C76; $15 \mathrm{~A} 18$.

Keywords: Spectrum of graphs, Eigenvalues of graphs, Graphs with exactly two non-negative eigenvalues.

Received: 01 Sep 2017, Accepted: 25 Oct 2017.

*Corresponding author

(C) 2017 Yazd University. 
as a graph whose its spectrum consists entirely of integers. For a graph $G, V(G)$ and $E(G)$ denote the vertex set and the edge set of $G$, respectively; $\bar{G}$ denotes the complement of $G$. The order of $G$ denotes the number of vertices of $G$. The closed neighborhood of a vertex $v$ of $G$ which is denoted by $N[v]$, is the set $\{u \in V(G): u v \in E(G)\} \cup\{v\}$. For every vertex $v \in V(G)$, the degree of $v$ is the number of edges incident with $v$. By $\delta(G)$ we mean the minimum degree of vertices of $G$. For two graphs $G$ and $H$ with disjoint vertex sets, $G+H$ denotes the graph with the vertex set $V(G) \cup V(H)$ and the edge set $E(G) \cup E(H)$, i.e. the disjoint union of two graphs $G$ and $H$. In particular, $n G$ denotes the disjoint union of $n$ copies of $G$. The complete product (join) $G \vee H$ of graphs $G$ and $H$ is the graph obtained from $G+H$ by joining every vertex of $G$ with every vertex of $H$. For positive integers $n_{1}, \ldots, n_{\ell}, K_{n_{1}, \ldots, n_{\ell}}$ denotes the complete multipartite graph with $\ell$ parts of sizes $n_{1}, \ldots, n_{\ell}$. Let $K_{n}, n K_{1}=\overline{K_{n}}, C_{n}$ and $P_{n}$ be the complete graph, the null graph, the cycle and the path on $n$ vertices, respectively.

It is well known that $\lambda_{1}(G)+\cdots+\lambda_{n}(G)=0$ and $\lambda_{1}^{2}(G)+\cdots+\lambda_{n}^{2}(G)=2 m$, where $m$ is the number of edges of $G$. Thus if $G$ has at least one edge, then $G$ has at least one positive eigenvalue. One of the attractive problems is the characterization of graphs with a few non-zero eigenvalues. In [I] all bipartite graphs with at most six non-zero eigenvalues have been characterized. The another interesting problem is the characterization of graphs with a few positive eigenvalues. In [5] Smith characterized all graphs with exactly one positive eigenvalue. In fact, a graph has exactly one positive eigenvalue if and only if its non-isolated vertices form a complete multipartite graph. Let $G$ be a graph with eigenvalues $\lambda_{1}(G) \geq \cdots \geq \lambda_{n}(G)$. In [4] Petrović has studied the characterization of graphs with exactly two non-negative eigenvalues. Recently in [Z] the author find all graphs $G$ with exactly two non-negative eigenvalues. In other words all graphs $G$ such that $\lambda_{1}(G) \geq 0, \lambda_{2}(G) \geq 0$ and $\lambda_{3}(G)<0$. It is proved that every graph $G$ with exactly two non-negative eigenvalues and with at least thirteen vertices has specific structure [2]. In this paper using computer we find all graphs $G$ such that $G$ has at most twelve vertices and $G$ has exactly two non-negative eigenvalues. More precisely we show that there are exactly 1575 connected graphs $G$ of order $n \leq 12$ such that $\lambda_{1}(G)>0, \lambda_{2}(G)>0$ and $\lambda_{3}(G)<0$. We investigate the integral graphs among these graphs. We find that among these 1575 graphs there are exactly two integral graphs.

\section{Graphs with exactly two non-negative eigenvalues}

In this section we state the structure of graphs $G$ that have exactly two non-negative eigenvalues. In other words the graphs $G$ with $\lambda_{3}(G)<0$. First we recall some definitions that are important for characterizing these graphs, see [2].

Definition 1.[2] For every integer $n \geq 2$, let $G_{n}$ be the graph of order $n$ such that $G_{n}$ is obtained from disjoint complete graphs $K_{\left\lceil\frac{n}{2}\right\rceil}$ and $K_{\left\lfloor\frac{n}{2}\right\rfloor}$ as following: Let $V\left(K_{\left\lceil\frac{n}{2}\right\rceil}\right)=\left\{v_{1}, \ldots, v_{\left\lceil\frac{n}{2}\right\rceil}\right\}$ and $V\left(K_{\left\lfloor\frac{n}{2}\right\rfloor}\right)=$ $\left\{w_{1}, \ldots, w_{\left\lfloor\frac{n}{2}\right\rfloor}\right\}$. Then add some new edges to $K_{\left\lceil\frac{n}{2}\right\rceil}+K_{\left\lfloor\frac{n}{2}\right\rfloor}$ such that the following hold: 
(i) $N\left[v_{1}\right] \subset \cdots \subset N\left[v_{\left\lceil\frac{n}{2}\right\rceil}\right]$ and $N\left[w_{1}\right] \subset \cdots \subset N\left[w_{\left\lfloor\frac{n}{2}\right]}\right]$.

(ii) $\left|N\left[v_{i}\right] \cap V\left(K_{\left\lfloor\frac{n}{2}\right\rfloor}\right)\right|=i-1$ for $i=1, \ldots,\left\lceil\frac{n}{2}\right\rceil$.

(iii) $\left|N\left[w_{j}\right] \cap V\left(K_{\left\lceil\frac{n}{2}\right\rceil}\right)\right|=\left\{\begin{array}{ll}j-1, & \text { if } n \text { is even; } \\ j, & \text { if } n \text { is odd }\end{array}\right.$ for $j=1, \ldots,\left\lfloor\frac{n}{2}\right\rfloor$.

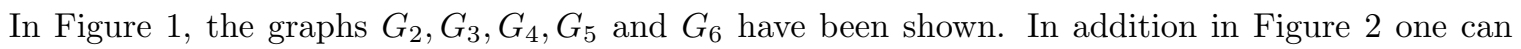
see the complement of $G_{7}, \ldots, G_{12}$. We remark that for every $n \geq 3, G_{n}$ is connected. We note that $G_{2 k}=B_{2 k}(1, \ldots, 1 ; 1, \ldots, 1)$ and $G_{2 k+1}=B_{2 k+1}(1, \ldots, 1 ; 1, \ldots, 1 ; 1)$, where $B_{2 k}$ and $B_{2 k+1}$ are the graphs that have been defined in [4].

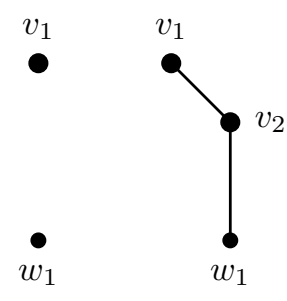

$G_{2}$

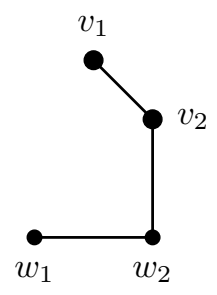

$G_{4}$

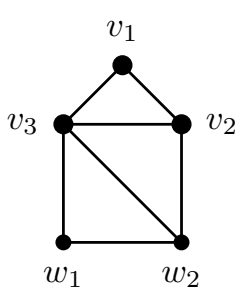

$G_{5}$

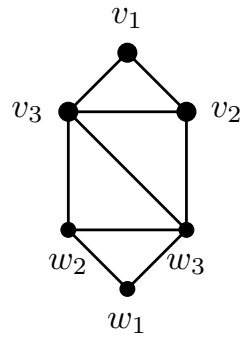

$G_{6}$

Figure 1. The graphs $G_{2}, G_{3}, G_{4}, G_{5}$ and $G_{6}$.

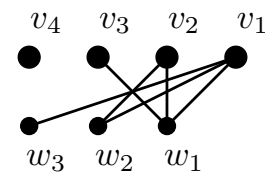

$\overline{G_{7}}$

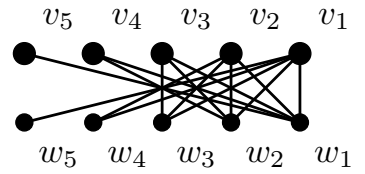

$\overline{G_{10}}$

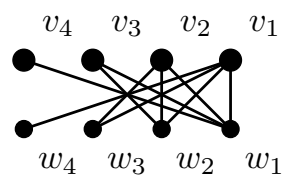

$\overline{G_{8}}$

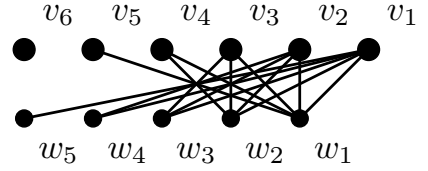

$\overline{G_{11}}$

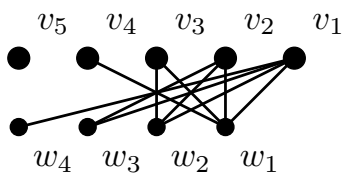

$\overline{G_{9}}$

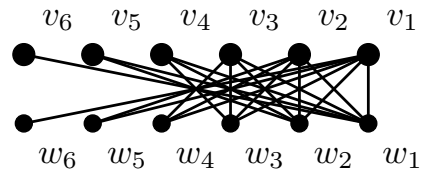

$\overline{G_{12}}$

Figure 2. The complement graphs of $G_{7}, G_{8}, G_{9}, G_{10}, G_{11}$ and $G_{12}$.

In fact $\lambda_{2}\left(G_{n}\right)>0$ and $\lambda_{3}\left(G_{n}\right)<0$ if and only if $4 \leq n \leq 12$, see [2].

Definition 2. [2] Let $G$ be a graph with vertex set $\left\{v_{1}, \ldots, v_{n}\right\}$. By $G\left[K_{t_{1}}, \ldots, K_{t_{n}}\right]$ we mean the graph obtained by replacing the vertex $v_{j}$ by the complete graph $K_{t_{j}}$ for $1 \leq j \leq n$, where every vertex of $K_{t_{i}}$ is adjacent to every vertex of $K_{t_{j}}$ if and only if $v_{i}$ is adjacent to $v_{j}$ (in $G$ ). For example $K_{2}\left[K_{p}, K_{q}\right] \cong K_{p+q}$ and $\overline{K_{2}}\left[K_{p}, K_{q}\right] \cong K_{p}+K_{q}$. 
One of the main results of $[2]$ is the following.

Theorem 1. [2] Let $G$ be a graph with eigenvalues $\lambda_{1} \geq \cdots \geq \lambda_{n}$. Assume that $\lambda_{3}<0$. Then the following hold:

(1) If $\lambda_{1}>0$ and $\lambda_{2}>0$, then $G \cong K_{p}+K_{q}$ for some integers $p, q \geq 2$ or there exist some positive integers $s$ and $t_{1}, \ldots, t_{s}$ so that $3 \leq s \leq 12$ and $t_{1}+\cdots+t_{s}=n$ and $G \cong G_{s}\left[K_{t_{1}}, \ldots, K_{t_{s}}\right]$.

(2) If $\lambda_{1}>0$ and $\lambda_{2}=0$, then $G \cong K_{1}+K_{n-1}$ or $G \cong K_{n} \backslash e$, where $e$ is an edge of $K_{n}$.

(3) If $\lambda_{1}>0$ and $\lambda_{2}<0$, then $G \cong K_{n}$.

The first part of Theorem $\mathbb{U}$ shows that to complete the characterization of the graphs with $\lambda_{3}<0$ it suffices to find all positive integers of $s$ and $t_{1}, \ldots, t_{s}$ so that $3 \leq s \leq 12$ and

$$
\lambda_{1}\left(G_{s}\left[K_{t_{1}}, \ldots, K_{t_{s}}\right]\right)>0, \lambda_{2}\left(G_{s}\left[K_{t_{1}}, \ldots, K_{t_{s}}\right]\right)>0 \text {, and } \lambda_{3}\left(G_{s}\left[K_{t_{1}}, \ldots, K_{t_{s}}\right]\right)<0 \text {. }
$$

We note that for $s \geq 3, G_{s}\left[K_{t_{1}}, \ldots, K_{t_{s}}\right]$ is connected. Also the number of its vertices is $t_{1}+\cdots+t_{s}$. In [] the author has found all values of $t_{1}, \ldots, t_{s}$ implicitly such that the Equation (四) holds. In fact in [2] it has been shown that every graph $G$ with at least thirteen vertices has special pattern. Here we study those graphs with at most twelve vertices. In fact we determine all positive integers $3 \leq s \leq 12$ and $t_{1}, \ldots, t_{s}$ such that $t_{1}+\cdots+t_{s} \leq 12$ and the Equation (四) holds. In other words we find all connected graphs $G$ with at most twelve vertices such that $\lambda_{2}>0$ and $\lambda_{3}<0$.

One can use the following result to compute the characteristic polynomial of $G_{s}\left[K_{t_{1}}, \ldots, K_{t_{s}}\right]$, the polynomial $P\left(G_{n}\left[K_{t_{1}}, \ldots, K_{t_{n}}\right], \lambda\right)$.

Theorem 2. [3] Let $n \geq 2$. Suppose that $\left\{v_{1}, \ldots, v_{n}\right\}$ is the vertex set of $G_{n}$ and $A=\left[a_{i j}\right]$ is the adjacency matrix of $G_{n}$ with respect to $\left\{v_{1}, \ldots, v_{n}\right\}\left(a_{i j}=1\right.$ if and only if $v_{i}$ and $v_{j}$ are adjacent and $a_{i j}=0$, otherwise). Let $t_{1}, \ldots, t_{n}$ be some positive integers and $M=\left[m_{i j}\right]$ be a $n \times n$ matrix, where

$$
m_{i j}:= \begin{cases}t_{i}-1, & \text { if } i=j \\ a_{i j} t_{j}, & \text { if } i \neq j .\end{cases}
$$

Then

$$
P\left(G_{n}\left[K_{t_{1}}, \ldots, K_{t_{n}}\right], \lambda\right)=(\lambda+1)^{t_{1}+\cdots+t_{n}-n} g(\lambda),
$$

where $g(\lambda)=\operatorname{det}(\lambda I-M)$. In addition, the multiplicity of -1 as an eigenvalue of $G_{n}\left[K_{t_{1}}, \ldots, K_{t_{n}}\right]$ is equal to $t_{1}+\cdots+t_{n}-n$.

\section{The list of all connected graphs with $\lambda_{2}>0$ and $\lambda_{3}<0$}

In this section we investigate the converse of the first part of Theorem $\mathbb{\square}$. We use Petrović's notation [4] that is very similar to the notation of Definition [2. We note that in Definition \&, the graph $G\left[H_{1}, \ldots, H_{n}\right]$ is dependent to the labeling of the vertices of $G$ while in the next definition first we fix a labeling for the vertices of $G_{n}$ ( see Definition प), and then use the operation of Definition घ. For 
Alg. Struc. Appl. Vol. 4 No. 1 (2017) 1-18.

instance we consider the labeling $v_{1}, \ldots, v_{s}$ and $w_{1}, \ldots, w_{s}$ for the vertices of $G_{2 s}$ and then apply the operation of Definition [.

Definition 3. [2] Let $s \geq 1$ be an integer and $n_{1}, \ldots, n_{2 s+1}$ be some positive integers. Let $B_{2 s}\left(n_{1}, \ldots, n_{s} ; n_{s+1}, \ldots, n_{2 s}\right)$ denote the graph obtained from $G_{2 s}$ by replacing the vertices $v_{1}$ by $K_{n_{1}}$, $v_{2}$ by $K_{n_{2}}, \ldots$, and $v_{s}$ by $K_{n_{s}}$ and $w_{1}$ by $K_{n_{s+1}}, w_{2}$ by $K_{n_{s+2}}, \ldots$, and $w_{s}$ by $K_{n_{2 s}}$ (see Definition ஐ). In other words

$$
B_{2 s}\left(n_{1}, \ldots, n_{s} ; n_{s+1}, \ldots, n_{2 s}\right)=G_{2 s}\left[K_{n_{1}}, \ldots, K_{n_{2 s}}\right],
$$

where the ordering of the vertices of $G_{2 s}$ is $V\left(G_{2 s}\right)=\left\{v_{1}, \ldots, v_{s}, w_{1}, \ldots, w_{s}\right\}$. Similarly, by $B_{2 s+1}\left(n_{1}, \ldots, n_{s} ; n_{s+1}, \ldots, n_{2 s} ; n_{2 s+1}\right)$ we mean

$$
B_{2 s+1}\left(n_{1}, \ldots, n_{s} ; n_{s+1}, \ldots, n_{2 s} ; n_{2 s+1}\right)=G_{2 s+1}\left[K_{n_{1}}, \ldots, K_{n_{2 s+1}}\right]
$$

where the ordering of the vertices of $G_{2 s+1}$ is $V\left(G_{2 s+1}\right)=\left\{v_{1}, \ldots, v_{s}, w_{1}, \ldots, w_{s}, v_{s+1}\right\}$, ( see Figure B).

Remark 3.1. [2] For every positive integers $s$ and $n_{1}, \ldots, n_{2 s+1}$, one can easily see that

$$
B_{2 s}\left(n_{1}, \ldots, n_{s} ; n_{s+1}, \ldots, n_{2 s}\right) \cong B_{2 s}\left(n_{s+1}, \ldots, n_{2 s} ; n_{1}, \ldots, n_{s}\right)
$$

and

$$
B_{2 s+1}\left(n_{1}, \ldots, n_{s} ; n_{s+1}, \ldots, n_{2 s} ; n_{2 s+1}\right) \cong B_{2 s+1}\left(n_{s+1}, \ldots, n_{2 s} ; n_{1}, \ldots, n_{s} ; n_{2 s+1}\right) .
$$

For avoiding the repeating, using the dictionary ordering on $\left(n_{1}, \ldots, n_{s}\right)$ and $\left(n_{s+1}, \ldots, n_{2 s}\right)$ we just cite one of the graphs $B_{2 s}\left(n_{1}, \ldots, n_{s} ; n_{s+1}, \ldots, n_{2 s}\right)$ or $B_{2 s}\left(n_{s+1}, \ldots, n_{2 s} ; n_{1}, \ldots, n_{s}\right)$ in our characterization. Similarly for the graphs $B_{2 s+1}\left(n_{1}, \ldots, n_{s} ; n_{s+1}, \ldots, n_{2 s} ; n_{2 s+1}\right)$ and

$B_{2 s+1}\left(n_{s+1}, \ldots, n_{2 s} ; n_{1}, \ldots, n_{s} ; n_{2 s+1}\right)$ we only consider one of them. For example since by dictionary ordering $(4,3,2) \geq(4,3,1)$ we use $B_{6}(4,3,2 ; 4,3,1)$ instead of $B_{6}(4,3,1 ; 4,3,2)$. As another example we use $B_{7}(5,3,2 ; 5,2,4 ; 8)$ instead of $B_{7}(5,2,4 ; 5,3,2 ; 8)$, since $(5,3,2) \geq(5,2,4)$.

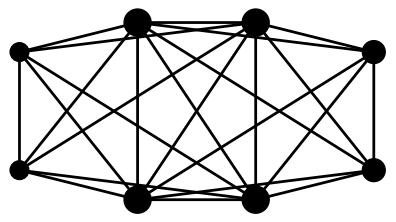

$B_{3}(2 ; 2 ; 4)$

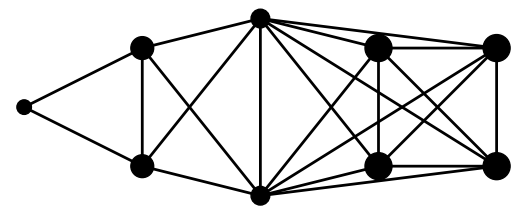

$B_{4}(1,2 ; 4,2)$

FIgURE 3 . The graphs $B_{3}(2 ; 2 ; 4)$ and $B_{4}(1,2 ; 4,2)$.

Now we are in a position to state the main result of the paper. Using Theorem $\square$ and computer one can check the the following.

Theorem 3. Let $G$ be a connected graph of order $n \leq 12$. Then $\lambda_{1}(G)>0, \lambda_{2}(G)>0$ and $\lambda_{3}(G)<0$ if and only if $G$ is isomorphic with one of the 1575 following graphs: 
(1) $B_{3}(2 ; 1 ; 1)$.

(2) $B_{3}(2 ; 1 ; 2), B_{3}(2 ; 2 ; 1), B_{3}(3 ; 1 ; 1)$.

(3) $B_{3}(2 ; 1 ; 3), B_{3}(2 ; 2 ; 2), B_{3}(3 ; 1 ; 2), B_{3}(3 ; 2 ; 1), B_{3}(4 ; 1 ; 1)$.

(4) $B_{3}(2 ; 1 ; 4), B_{3}(2 ; 2 ; 3), B_{3}(3 ; 1 ; 3), B_{3}(3 ; 2 ; 2), B_{3}(3 ; 3 ; 1), B_{3}(4 ; 1 ; 2), B_{3}(4 ; 2 ; 1), B_{3}(5 ; 1 ; 1)$.

(5) $B_{3}(2 ; 1 ; 5), B_{3}(2 ; 2 ; 4), B_{3}(3 ; 1 ; 4), B_{3}(3 ; 2 ; 3), B_{3}(3 ; 3 ; 2), B_{3}(4 ; 1 ; 3), B_{3}(4 ; 2 ; 2), B_{3}(4 ; 3 ; 1)$.

(6) $B_{3}(5 ; 1 ; 2), B_{3}(5 ; 2 ; 1), B_{3}(6 ; 1 ; 1)$.

(7) $B_{3}(2 ; 1 ; 6), B_{3}(2 ; 2 ; 5), B_{3}(3 ; 1 ; 5), B_{3}(3 ; 2 ; 4), B_{3}(3 ; 3 ; 3), B_{3}(4 ; 1 ; 4), B_{3}(4 ; 2 ; 3), B_{3}(4 ; 3 ; 2)$.

(8) $B_{3}(4 ; 4 ; 1), B_{3}(5 ; 1 ; 3), B_{3}(5 ; 2 ; 2), B_{3}(5 ; 3 ; 1), B_{3}(6 ; 1 ; 2), B_{3}(6 ; 2 ; 1), B_{3}(7 ; 1 ; 1)$.

(9) $B_{3}(2 ; 1 ; 7), B_{3}(2 ; 2 ; 6), B_{3}(3 ; 1 ; 6), B_{3}(3 ; 2 ; 5), B_{3}(3 ; 3 ; 4), B_{3}(4 ; 1 ; 5), B_{3}(4 ; 2 ; 4), B_{3}(4 ; 3 ; 3)$.

(10) $B_{3}(4 ; 4 ; 2), B_{3}(5 ; 1 ; 4), B_{3}(5 ; 2 ; 3), B_{3}(5 ; 3 ; 2), B_{3}(5 ; 4 ; 1), B_{3}(6 ; 1 ; 3), B_{3}(6 ; 2 ; 2), B_{3}(6 ; 3 ; 1)$.

(11) $B_{3}(7 ; 1 ; 2), B_{3}(7 ; 2 ; 1), B_{3}(8 ; 1 ; 1)$.

(12) $B_{3}(2 ; 1 ; 8), B_{3}(2 ; 2 ; 7), B_{3}(3 ; 1 ; 7), B_{3}(3 ; 2 ; 6), B_{3}(3 ; 3 ; 5), B_{3}(4 ; 1 ; 6), B_{3}(4 ; 2 ; 5), B_{3}(4 ; 3 ; 4)$.

(13) $B_{3}(4 ; 4 ; 3), B_{3}(5 ; 1 ; 5), B_{3}(5 ; 2 ; 4), B_{3}(5 ; 3 ; 3), B_{3}(5 ; 4 ; 2), B_{3}(5 ; 5 ; 1), B_{3}(6 ; 1 ; 4), B_{3}(6 ; 2 ; 3)$.

(14) $B_{3}(6 ; 3 ; 2), B_{3}(6 ; 4 ; 1), B_{3}(7 ; 1 ; 3), B_{3}(7 ; 2 ; 2), B_{3}(7 ; 3 ; 1), B_{3}(8 ; 1 ; 2), B_{3}(8 ; 2 ; 1), B_{3}(9 ; 1 ; 1)$.

(15) $B_{3}(2 ; 1 ; 9), B_{3}(2 ; 2 ; 8), B_{3}(3 ; 1 ; 8), B_{3}(3 ; 2 ; 7), B_{3}(3 ; 3 ; 6), B_{3}(4 ; 1 ; 7), B_{3}(4 ; 2 ; 6), B_{3}(4 ; 3 ; 5)$.

(16) $B_{3}(4 ; 4 ; 4), B_{3}(5 ; 1 ; 6), B_{3}(5 ; 2 ; 5), B_{3}(5 ; 3 ; 4), B_{3}(5 ; 4 ; 3), B_{3}(5 ; 5 ; 2), B_{3}(6 ; 1 ; 5), B_{3}(6 ; 2 ; 4)$.

(17) $B_{3}(6 ; 3 ; 3), B_{3}(6 ; 4 ; 2), B_{3}(6 ; 5 ; 1), B_{3}(7 ; 1 ; 4), B_{3}(7 ; 2 ; 3), B_{3}(7 ; 3 ; 2), B_{3}(7 ; 4 ; 1), B_{3}(8 ; 1 ; 3)$.

(18) $B_{3}(8 ; 2 ; 2), B_{3}(8 ; 3 ; 1), B_{3}(9 ; 1 ; 2), B_{3}(9 ; 2 ; 1), B_{3}(10 ; 1 ; 1)$.

(19) $B_{4}(1,1 ; 1,1)$.

(20) $B_{4}(1,2 ; 1,1), B_{4}(2,1 ; 1,1)$.

(21) $B_{4}(1,2 ; 1,2), B_{4}(1,3 ; 1,1), B_{4}(2,1 ; 1,2), B_{4}(2,1 ; 2,1), B_{4}(2,2 ; 1,1), B_{4}(3,1 ; 1,1)$.

(22) $B_{4}(1,3 ; 1,2), B_{4}(1,4 ; 1,1), B_{4}(2,1 ; 1,3), B_{4}(2,2 ; 1,2), B_{4}(2,2 ; 2,1)$.

(23) $B_{4}(2,3 ; 1,1), B_{4}(3,1 ; 1,2), B_{4}(3,1 ; 2,1), B_{4}(3,2 ; 1,1), B_{4}(4,1 ; 1,1)$.

(24) $B_{4}(1,3 ; 1,3), B_{4}(1,4 ; 1,2), B_{4}(1,5 ; 1,1), B_{4}(2,1 ; 1,4), B_{4}(2,2 ; 1,3), B_{4}(2,2 ; 2,2), B_{4}(2,3 ; 1,2)$.

$(25) B_{4}(2,3 ; 2,1), B_{4}(2,4 ; 1,1), B_{4}(3,1 ; 1,3), B_{4}(3,1 ; 2,2), B_{4}(3,1 ; 3,1), B_{4}(3,2 ; 1,2), B_{4}(3,2 ; 2,1)$.

(26) $B_{4}(3,3 ; 1,1), B_{4}(4,1 ; 1,2), B_{4}(4,1 ; 2,1), B_{4}(4,2 ; 1,1), B_{4}(5,1 ; 1,1)$.

(27) $B_{4}(1,4 ; 1,3), B_{4}(1,5 ; 1,2), B_{4}(1,6 ; 1,1), B_{4}(2,1 ; 1,5), B_{4}(2,2 ; 1,4), B_{4}(2,3 ; 1,3), B_{4}(2,3 ; 2,2)$.

(28) $B_{4}(2,4 ; 1,2), B_{4}(2,4 ; 2,1), B_{4}(2,5 ; 1,1), B_{4}(3,1 ; 1,4), B_{4}(3,1 ; 2,3), B_{4}(3,2 ; 1,3), B_{4}(3,2 ; 2,2)$.

(29) $B_{4}(3,2 ; 3,1), B_{4}(3,3 ; 1,2), B_{4}(3,3 ; 2,1), B_{4}(3,4 ; 1,1), B_{4}(4,1 ; 1,3), B_{4}(4,1 ; 2,2), B_{4}(4,1 ; 3,1)$.

(30) $B_{4}(4,2 ; 1,2), B_{4}(4,2 ; 2,1), B_{4}(4,3 ; 1,1), B_{4}(5,1 ; 1,2), B_{4}(5,1 ; 2,1), B_{4}(5,2 ; 1,1), B_{4}(6,1 ; 1,1)$.

(31) $B_{4}(1,4 ; 1,4), B_{4}(1,5 ; 1,3), B_{4}(1,6 ; 1,2), B_{4}(1,7 ; 1,1), B_{4}(2,1 ; 1,6), B_{4}(2,2 ; 1,5), B_{4}(2,3 ; 1,4)$.

(32) $B_{4}(2,3 ; 2,3), B_{4}(2,4 ; 1,3), B_{4}(2,4 ; 2,2), B_{4}(2,5 ; 1,2), B_{4}(2,5 ; 2,1), B_{4}(2,6 ; 1,1), B_{4}(3,1 ; 1,5)$.

(33) $B_{4}(3,1 ; 2,4), B_{4}(3,2 ; 1,4), B_{4}(3,2 ; 2,3), B_{4}(3,3 ; 1,3), B_{4}(3,3 ; 2,2), B_{4}(3,3 ; 3,1), B_{4}(3,4 ; 1,2)$.

(34) $B_{4}(3,4 ; 2,1), B_{4}(3,5 ; 1,1), B_{4}(4,1 ; 1,4), B_{4}(4,1 ; 2,3), B_{4}(4,1 ; 3,2), B_{4}(4,1 ; 4,1), B_{4}(4,2 ; 1,3)$.

(35) $B_{4}(4,2 ; 2,2), B_{4}(4,2 ; 3,1), B_{4}(4,3 ; 1,2), B_{4}(4,3 ; 2,1), B_{4}(4,4 ; 1,1), B_{4}(5,1 ; 1,3), B_{4}(5,1 ; 2,2)$.

(36) $B_{4}(5,1 ; 3,1), B_{4}(5,2 ; 1,2), B_{4}(5,2 ; 2,1), B_{4}(5,3 ; 1,1), B_{4}(6,1 ; 1,2), B_{4}(6,1 ; 2,1), B_{4}(6,2 ; 1,1)$. 
(37) $B_{4}(7,1 ; 1,1)$.

(38) $B_{4}(1,5 ; 1,4), B_{4}(1,6 ; 1,3), B_{4}(1,7 ; 1,2), B_{4}(1,8 ; 1,1), B_{4}(2,1 ; 1,7), B_{4}(2,2 ; 1,6), B_{4}(2,3 ; 1,5)$.

(39) $B_{4}(2,4 ; 1,4), B_{4}(2,4 ; 2,3), B_{4}(2,5 ; 1,3), B_{4}(2,5 ; 2,2), B_{4}(2,6 ; 1,2), B_{4}(2,6 ; 2,1), B_{4}(2,7 ; 1,1)$.

(40) $B_{4}(3,1 ; 1,6), B_{4}(3,1 ; 2,5), B_{4}(3,2 ; 1,5), B_{4}(3,2 ; 2,4), B_{4}(3,3 ; 1,4), B_{4}(3,3 ; 2,3), B_{4}(3,4 ; 1,3)$.

(41) $B_{4}(3,4 ; 2,2), B_{4}(3,4 ; 3,1), B_{4}(3,5 ; 1,2), B_{4}(3,5 ; 2,1), B_{4}(3,6 ; 1,1), B_{4}(4,1 ; 1,5), B_{4}(4,1 ; 2,4)$.

(42) $B_{4}(4,1 ; 3,3), B_{4}(4,2 ; 1,4), B_{4}(4,2 ; 2,3), B_{4}(4,2 ; 4,1), B_{4}(4,3 ; 1,3), B_{4}(4,4 ; 1,2), B_{4}(4,4 ; 2,1)$.

(43) $B_{4}(4,5 ; 1,1), B_{4}(5,1 ; 1,4), B_{4}(5,1 ; 2,3), B_{4}(5,1 ; 3,2), B_{4}(5,1 ; 4,1), B_{4}(5,2 ; 1,3), B_{4}(5,2 ; 2,2)$.

(44) $B_{4}(5,2 ; 3,1), B_{4}(5,3 ; 1,2), B_{4}(5,3 ; 2,1), B_{4}(5,4 ; 1,1), B_{4}(6,1 ; 1,3), B_{4}(6,1 ; 2,2), B_{4}(6,1 ; 3,1)$.

(45) $B_{4}(6,2 ; 1,2), B_{4}(6,2 ; 2,1), B_{4}(6,3 ; 1,1), B_{4}(7,1 ; 1,2), B_{4}(7,1 ; 2,1), B_{4}(7,2 ; 1,1), B_{4}(8,1 ; 1,1)$.

(46) $B_{4}(1,5 ; 1,5), B_{4}(1,6 ; 1,4), B_{4}(1,7 ; 1,3), B_{4}(1,8 ; 1,2), B_{4}(1,9 ; 1,1), B_{4}(2,1 ; 1,8), B_{4}(2,2 ; 1,7)$.

(47) $B_{4}(2,3 ; 1,6), B_{4}(2,4 ; 1,5), B_{4}(2,4 ; 2,4), B_{4}(2,5 ; 1,4), B_{4}(2,5 ; 2,3), B_{4}(2,6 ; 1,3), B_{4}(2,6 ; 2,2)$.

(48) $B_{4}(2,7 ; 1,2), B_{4}(2,7 ; 2,1), B_{4}(2,8 ; 1,1), B_{4}(3,1 ; 1,7), B_{4}(3,1 ; 2,6), B_{4}(3,2 ; 1,6), B_{4}(3,2 ; 2,5)$.

(49) $B_{4}(3,3 ; 1,5), B_{4}(3,3 ; 2,4), B_{4}(3,4 ; 1,4), B_{4}(3,5 ; 1,3), B_{4}(3,5 ; 2,2), B_{4}(3,5 ; 3,1), B_{4}(3,6 ; 1,2)$.

(50) $B_{4}(3,6 ; 2,1), B_{4}(3,7 ; 1,1), B_{4}(4,1 ; 1,6), B_{4}(4,1 ; 2,5), B_{4}(4,2 ; 1,5), B_{4}(4,2 ; 2,4), B_{4}(4,3 ; 1,4)$.

(51) $B_{4}(4,4 ; 1,3), B_{4}(4,5 ; 1,2), B_{4}(4,5 ; 2,1), B_{4}(4,6 ; 1,1), B_{4}(5,1 ; 1,5), B_{4}(5,1 ; 2,4), B_{4}(5,1 ; 3,3)$.

(52) $B_{4}(5,1 ; 4,2), B_{4}(5,1 ; 5,1), B_{4}(5,2 ; 1,4), B_{4}(5,3 ; 1,3), B_{4}(5,4 ; 1,2), B_{4}(5,5 ; 1,1), B_{4}(6,1 ; 1,4)$.

(53) $B_{4}(6,1 ; 2,3), B_{4}(6,1 ; 3,2), B_{4}(6,1 ; 4,1), B_{4}(6,2 ; 1,3), B_{4}(6,2 ; 2,2), B_{4}(6,2 ; 3,1), B_{4}(6,3 ; 1,2)$.

(54) $B_{4}(6,3 ; 2,1), B_{4}(6,4 ; 1,1), B_{4}(7,1 ; 1,3), B_{4}(7,1 ; 2,2), B_{4}(7,1 ; 3,1), B_{4}(7,2 ; 1,2), B_{4}(7,2 ; 2,1)$.

(55) $B_{4}(7,3 ; 1,1), B_{4}(8,1 ; 1,2), B_{4}(8,1 ; 2,1), B_{4}(8,2 ; 1,1), B_{4}(9,1 ; 1,1)$.

(56) $B_{5}(1,1 ; 1,1 ; 1)$.

(57) $B_{5}(1,1 ; 1,1 ; 2), B_{5}(1,2 ; 1,1 ; 1), B_{5}(2,1 ; 1,1 ; 1)$.

(58) $B_{5}(1,1 ; 1,1 ; 3), B_{5}(1,2 ; 1,1 ; 2), B_{5}(1,2 ; 1,2 ; 1), B_{5}(1,3 ; 1,1 ; 1), B_{5}(2,1 ; 1,1 ; 2), B_{5}(2,1 ; 1,2 ; 1)$.

(59) $B_{5}(2,1 ; 2,1 ; 1), B_{5}(2,2 ; 1,1 ; 1), B_{5}(3,1 ; 1,1 ; 1)$.

(60) $B_{5}(1,1 ; 1,1 ; 4), B_{5}(1,2 ; 1,1 ; 3), B_{5}(1,2 ; 1,2 ; 2), B_{5}(1,3 ; 1,1 ; 2), B_{5}(1,3 ; 1,2 ; 1), B_{5}(1,4 ; 1,1 ; 1)$.

(61) $B_{5}(2,1 ; 1,1 ; 3), B_{5}(2,1 ; 1,2 ; 2), B_{5}(2,1 ; 1,3 ; 1), B_{5}(2,1 ; 2,1 ; 2), B_{5}(2,2 ; 1,1 ; 2), B_{5}(2,2 ; 1,2 ; 1)$.

(62) $B_{5}(2,2 ; 2,1 ; 1), B_{5}(2,3 ; 1,1 ; 1), B_{5}(3,1 ; 1,1 ; 2), B_{5}(3,1 ; 1,2 ; 1), B_{5}(3,1 ; 2,1 ; 1), B_{5}(3,2 ; 1,1 ; 1)$.

(63) $B_{5}(4,1 ; 1,1 ; 1)$.

(64) $B_{5}(1,1 ; 1,1 ; 5), B_{5}(1,2 ; 1,1 ; 4), B_{5}(1,2 ; 1,2 ; 3), B_{5}(1,3 ; 1,1 ; 3), B_{5}(1,3 ; 1,2 ; 2), B_{5}(1,3 ; 1,3 ; 1)$.

(65) $B_{5}(1,4 ; 1,1 ; 2), B_{5}(1,4 ; 1,2 ; 1), B_{5}(1,5 ; 1,1 ; 1), B_{5}(2,1 ; 1,1 ; 4), B_{5}(2,1 ; 1,2 ; 3), B_{5}(2,1 ; 1,3 ; 2)$.

(66) $B_{5}(2,1 ; 1,4 ; 1), B_{5}(2,1 ; 2,1 ; 3), B_{5}(2,2 ; 1,1 ; 3), B_{5}(2,2 ; 1,2 ; 2), B_{5}(2,2 ; 1,3 ; 1), B_{5}(2,2 ; 2,1 ; 2)$.

(67) $B_{5}(2,3 ; 1,1 ; 2), B_{5}(2,3 ; 1,2 ; 1), B_{5}(2,3 ; 2,1 ; 1), B_{5}(2,4 ; 1,1 ; 1), B_{5}(3,1 ; 1,1 ; 3), B_{5}(3,1 ; 1,2 ; 2)$.

(68) $B_{5}(3,1 ; 1,3 ; 1), B_{5}(3,1 ; 2,1 ; 2), B_{5}(3,1 ; 2,2 ; 1), B_{5}(3,1 ; 3,1 ; 1), B_{5}(3,2 ; 1,1 ; 2), B_{5}(3,2 ; 1,2 ; 1)$.

(69) $B_{5}(3,2 ; 2,1 ; 1), B_{5}(3,3 ; 1,1,1), B_{5}(4,1 ; 1,1 ; 2), B_{5}(4,1 ; 1,2 ; 1), B_{5}(4,1 ; 2,1 ; 1), B_{5}(4,2 ; 1,1 ; 1)$.

(70) $B_{5}(5,1 ; 1,1 ; 1)$.

(71) $B_{5}(1,1 ; 1,1 ; 6), B_{5}(1,2 ; 1,1 ; 5), B_{5}(1,2 ; 1,2 ; 4), B_{5}(1,3 ; 1,1 ; 4), B_{5}(1,3 ; 1,2 ; 3), B_{5}(1,3 ; 1,3 ; 2)$.

(72) $B_{5}(1,4 ; 1,1 ; 3), B_{5}(1,4 ; 1,2 ; 2), B_{5}(1,4 ; 1,3,1), B_{5}(1,5 ; 1,1 ; 2), B_{5}(1,5 ; 1,2 ; 1), B_{5}(1,6 ; 1,1 ; 1)$. 
(73) $B_{5}(2,1 ; 1,1 ; 5), B_{5}(2,1 ; 1,2 ; 4), B_{5}(2,1 ; 1,3 ; 3), B_{5}(2,1 ; 1,4 ; 2), B_{5}(2,1 ; 1,5 ; 1), B_{5}(2,1 ; 2,1 ; 4)$.

(74) $B_{5}(2,2 ; 1,1 ; 4), B_{5}(2,2 ; 1,2 ; 3), B_{5}(2,2 ; 1,3 ; 2), B_{5}(2,2 ; 1,4 ; 1), B_{5}(2,2 ; 2,1 ; 3), B_{5}(2,3 ; 1,1 ; 3)$.

(75) $B_{5}(2,3 ; 1,3 ; 1), B_{5}(2,3 ; 2,1 ; 2), B_{5}(2,4 ; 1,1 ; 2), B_{5}(2,4 ; 1,2 ; 1), B_{5}(2,4 ; 2,1 ; 1), B_{5}(2,5 ; 1,1 ; 1)$.

(76) $B_{5}(3,1 ; 1,1 ; 4), B_{5}(3,1 ; 1,2 ; 3), B_{5}(3,1 ; 1,3 ; 2), B_{5}(3,1 ; 1,4 ; 1), B_{5}(3,1 ; 2,1 ; 3), B_{5}(3,1 ; 2,2 ; 2)$.

(77) $B_{5}(3,1 ; 2,3 ; 1), B_{5}(3,1 ; 3,1 ; 2), B_{5}(3,2 ; 1,1 ; 3), B_{5}(3,2 ; 1,2 ; 2), B_{5}(3,2 ; 1,3 ; 1), B_{5}(3,2 ; 2,1 ; 2)$.

(78) $B_{5}(3,2 ; 3,1 ; 1), B_{5}(3,3 ; 1,1 ; 2), B_{5}(3,3 ; 1,2 ; 1), B_{5}(3,4 ; 1,1 ; 1), B_{5}(4,1 ; 1,1 ; 3), B_{5}(4,1 ; 1,2 ; 2)$.

(79) $B_{5}(4,1 ; 1,3 ; 1), B_{5}(4,1 ; 2,1 ; 2), B_{5}(4,1 ; 2,2 ; 1), B_{5}(4,1 ; 3,1 ; 1), B_{5}(4,2 ; 1,1 ; 2), B_{5}(4,2 ; 1,2 ; 1)$.

(80) $B_{5}(4,2 ; 2,1 ; 1), B_{5}(4,3 ; 1,1 ; 1), B_{5}(5,1 ; 1,1 ; 2), B_{5}(5,1 ; 1,2 ; 1), B_{5}(5,1 ; 2,1 ; 1), B_{5}(5,2 ; 1,1 ; 1)$.

(81) $B_{5}(6,1 ; 1,1 ; 1)$.

(82) $B_{5}(1,1 ; 1,1 ; 7), B_{5}(1,2 ; 1,1 ; 6), B_{5}(1,2 ; 1,2 ; 5), B_{5}(1,3 ; 1,1 ; 5), B_{5}(1,3 ; 1,2 ; 4), B_{5}(1,4 ; 1,1 ; 4)$.

(83) $B_{5}(1,4 ; 1,2 ; 3), B_{5}(1,4 ; 1,3 ; 2), B_{5}(1,4 ; 1,4 ; 1), B_{5}(1,5 ; 1,1 ; 3), B_{5}(1,5 ; 1,2 ; 2), B_{5}(1,5 ; 1,3 ; 1)$.

(84) $B_{5}(1,6 ; 1,1 ; 2), B_{5}(1,6 ; 1,2 ; 1), B_{5}(1,7 ; 1,1 ; 1), B_{5}(2,1 ; 1,1 ; 6), B_{5}(2,1 ; 1,2 ; 5), B_{5}(2,1 ; 1,3 ; 4)$.

(85) $B_{5}(2,1 ; 1,4 ; 3), B_{5}(2,1 ; 1,5 ; 2), B_{5}(2,1 ; 1,6 ; 1), B_{5}(2,1 ; 2,1 ; 5), B_{5}(2,2 ; 1,1 ; 5), B_{5}(2,2 ; 1,2 ; 4)$.

(86) $B_{5}(2,2 ; 1,4 ; 2), B_{5}(2,2 ; 1,5 ; 1), B_{5}(2,2 ; 2,1 ; 4), B_{5}(2,3 ; 1,1 ; 4), B_{5}(2,3 ; 1,4 ; 1), B_{5}(2,3 ; 2,1 ; 3)$.

(87) $B_{5}(2,4 ; 1,1 ; 3), B_{5}(2,4 ; 1,3 ; 1), B_{5}(2,5 ; 1,1 ; 2), B_{5}(2,5 ; 1,2 ; 1), B_{5}(2,5 ; 2,1 ; 1), B_{5}(2,6 ; 1,1 ; 1)$.

(88) $B_{5}(3,1 ; 1,1 ; 5), B_{5}(3,1 ; 1,2 ; 4), B_{5}(3,1 ; 1,3 ; 3), B_{5}(3,1 ; 1,4 ; 2), B_{5}(3,1 ; 1,5 ; 1), B_{5}(3,1 ; 2,1 ; 4)$.

(89) $B_{5}(3,1 ; 2,2 ; 3), B_{5}(3,1 ; 2,3 ; 2), B_{5}(3,1 ; 2,4 ; 1), B_{5}(3,1 ; 3,1 ; 3), B_{5}(3,2 ; 1,1 ; 4), B_{5}(3,2 ; 1,2 ; 3)$.

(90) $B_{5}(3,2 ; 1,3 ; 2), B_{5}(3,2 ; 1,4 ; 1), B_{5}(3,2 ; 2,1 ; 3), B_{5}(3,2 ; 3,1 ; 2), B_{5}(3,3 ; 1,1 ; 3), B_{5}(3,3 ; 1,3 ; 1)$.

(91) $B_{5}(3,5 ; 1,1 ; 1), B_{5}(4,1 ; 1,1 ; 4), B_{5}(4,1 ; 1,2 ; 3), B_{5}(4,1 ; 1,3 ; 2), B_{5}(4,1 ; 1,4 ; 1), B_{5}(4,1 ; 2,1 ; 3)$.

(92) $B_{5}(4,1 ; 2,2 ; 2), B_{5}(4,1 ; 2,3 ; 1), B_{5}(4,1 ; 3,1 ; 2), B_{5}(4,1 ; 3,2 ; 1), B_{5}(4,1 ; 4,1 ; 1), B_{5}(4,2 ; 1,1 ; 3)$.

(93) $B_{5}(4,2 ; 1,2 ; 2), B_{5}(4,2 ; 1,3 ; 1), B_{5}(4,2 ; 2,1 ; 2), B_{5}(4,3 ; 1,1 ; 2), B_{5}(4,3 ; 1,2 ; 1), B_{5}(4,4 ; 1,1 ; 1)$.

(94) $B_{5}(5,1 ; 1,1 ; 3), B_{5}(5,1 ; 1,2 ; 2), B_{5}(5,1 ; 1,3 ; 1), B_{5}(5,1 ; 2,1 ; 2), B_{5}(5,1 ; 2,2 ; 1), B_{5}(5,1 ; 3,1 ; 1)$.

(95) $B_{5}(5,2 ; 1,1 ; 2), B_{5}(5,2 ; 1,2 ; 1), B_{5}(5,2 ; 2,1 ; 1), B_{5}(5,3 ; 1,1 ; 1), B_{5}(6,1 ; 1,1 ; 2), B_{5}(6,1 ; 1,2 ; 1)$.

(96) $B_{5}(6,1 ; 2,1 ; 1), B_{5}(6,2 ; 1,1 ; 1), B_{5}(7,1 ; 1,1 ; 1)$.

(97) $B_{5}(1,1 ; 1,1 ; 8), B_{5}(1,2 ; 1,1 ; 7), B_{5}(1,2 ; 1,2 ; 6), B_{5}(1,3 ; 1,1 ; 6), B_{5}(1,3 ; 1,2 ; 5), B_{5}(1,4 ; 1,1 ; 5)$.

(98) $B_{5}(1,5 ; 1,1 ; 4), B_{5}(1,5 ; 1,2 ; 3), B_{5}(1,5 ; 1,3 ; 2), B_{5}(1,5 ; 1,4 ; 1), B_{5}(1,6 ; 1,1 ; 3), B_{5}(1,6 ; 1,2 ; 2)$.

(99) $B_{5}(1,6 ; 1,3 ; 1), B_{5}(1,7 ; 1,1 ; 2), B_{5}(1,7 ; 1,2 ; 1), B_{5}(1,8 ; 1,1 ; 1), B_{5}(2,1 ; 1,1 ; 7), B_{5}(2,1 ; 1,2 ; 6)$.

(100) $B_{5}(2,1 ; 1,3 ; 5), B_{5}(2,1 ; 1,4 ; 4), B_{5}(2,1 ; 1,5 ; 3), B_{5}(2,1 ; 1,6 ; 2), B_{5}(2,1 ; 1,7 ; 1), B_{5}(2,1 ; 2,1 ; 6)$.

(101) $B_{5}(2,2 ; 1,1 ; 6), B_{5}(2,2 ; 1,2 ; 5), B_{5}(2,2 ; 1,5 ; 2), B_{5}(2,2 ; 1,6 ; 1), B_{5}(2,2 ; 2,1 ; 5), B_{5}(2,3 ; 1,1 ; 5)$.

(102) $B_{5}(2,3 ; 1,5 ; 1), B_{5}(2,3 ; 2,1 ; 4), B_{5}(2,4 ; 1,1 ; 4), B_{5}(2,4 ; 1,4 ; 1), B_{5}(2,6 ; 1,1 ; 2), B_{5}(2,6 ; 1,2 ; 1)$.

(103) $B_{5}(2,6 ; 2,1 ; 1), B_{5}(2,7 ; 1,1 ; 1), B_{5}(3,1 ; 1,1 ; 6), B_{5}(3,1 ; 1,2 ; 5), B_{5}(3,1 ; 1,3 ; 4), B_{5}(3,1 ; 1,4 ; 3)$.

(104) $B_{5}(3,1 ; 1,5 ; 2), B_{5}(3,1 ; 1,6 ; 1), B_{5}(3,1 ; 2,1 ; 5), B_{5}(3,1 ; 2,2 ; 4), B_{5}(3,1 ; 3,1 ; 4), B_{5}(3,2 ; 1,1 ; 5)$.

(105) $B_{5}(3,2 ; 1,5 ; 1), B_{5}(3,2 ; 2,1 ; 4), B_{5}(3,2 ; 3,1 ; 3), B_{5}(3,3 ; 1,1 ; 4), B_{5}(3,3 ; 1,4 ; 1), B_{5}(3,6 ; 1,1 ; 1)$.

(106) $B_{5}(4,1 ; 1,1 ; 5), B_{5}(4,1 ; 1,2 ; 4), B_{5}(4,1 ; 1,3 ; 3), B_{5}(4,1 ; 1,4 ; 2), B_{5}(4,1 ; 1,5 ; 1), B_{5}(4,1 ; 2,1 ; 4)$.

(107) $B_{5}(4,1 ; 2,2 ; 3), B_{5}(4,1 ; 2,3 ; 2), B_{5}(4,1 ; 2,4 ; 1), B_{5}(4,1 ; 3,1 ; 3), B_{5}(4,1 ; 4,1 ; 2), B_{5}(4,2 ; 1,1 ; 4)$.

(108) $B_{5}(4,2 ; 1,2 ; 3), B_{5}(4,2 ; 1,3 ; 2), B_{5}(4,2 ; 1,4 ; 1), B_{5}(4,2 ; 2,1 ; 3), B_{5}(5,1 ; 1,1 ; 4), B_{5}(5,1 ; 1,2 ; 3)$. 
(109) $B_{5}(5,1 ; 1,3 ; 2), B_{5}(5,1 ; 1,4 ; 1), B_{5}(5,1 ; 2,1 ; 3), B_{5}(5,1 ; 2,2 ; 2), B_{5}(5,1 ; 2,3 ; 1), B_{5}(5,1 ; 3,1 ; 2)$.

(110) $B_{5}(5,1 ; 3,2 ; 1), B_{5}(5,1 ; 4,1 ; 1), B_{5}(5,2 ; 1,1 ; 3), B_{5}(5,2 ; 1,2 ; 2), B_{5}(5,2 ; 1,3 ; 1), B_{5}(5,3 ; 1,1 ; 2)$.

(111) $B_{5}(5,3 ; 1,2 ; 1), B_{5}(5,4 ; 1,1 ; 1), B_{5}(6,1 ; 1,1 ; 3), B_{5}(6,1 ; 1,2 ; 2), B_{5}(6,1 ; 1,3 ; 1), B_{5}(6,1 ; 2,1 ; 2)$.

(112) $B_{5}(6,1 ; 2,2 ; 1), B_{5}(6,1 ; 3,1 ; 1), B_{5}(6,2 ; 1,1 ; 2), B_{5}(6,2 ; 1,2 ; 1), B_{5}(6,2 ; 2,1 ; 1), B_{5}(6,3 ; 1,1 ; 1)$.

(113) $B_{5}(7,1 ; 1,1 ; 2), B_{5}(7,1 ; 1,2 ; 1), B_{5}(7,1 ; 2,1 ; 1), B_{5}(7,2 ; 1,1 ; 1), B_{5}(8,1 ; 1,1 ; 1)$.

(114) $B_{6}(1,1,1 ; 1,1,1)$.

(115) $B_{6}(1,1,2 ; 1,1,1), B_{6}(1,2,1 ; 1,1,1), B_{6}(2,1,1 ; 1,1,1)$.

(116) $B_{6}(1,1,2 ; 1,1,2), B_{6}(1,1,3 ; 1,1,1), B_{6}(1,2,1 ; 1,1,2), B_{6}(1,2,1 ; 1,2,1), B_{6}(1,2,2 ; 1,1,1)$.

(117) $B_{6}(1,3,1 ; 1,1,1), B_{6}(2,1,1 ; 1,1,2), B_{6}(2,1,1 ; 1,2,1), B_{6}(2,1,1 ; 2,1,1), B_{6}(2,1,2 ; 1,1,1)$.

(118) $B_{6}(2,2,1 ; 1,1,1), B_{6}(3,1,1 ; 1,1,1)$.

(119) $B_{6}(1,1,3 ; 1,1,2), B_{6}(1,1,4 ; 1,1,1), B_{6}(1,2,1 ; 1,1,3), B_{6}(1,2,2 ; 1,1,2), B_{6}(1,2,2 ; 1,2,1)$.

(120) $B_{6}(1,2,3 ; 1,1,1), B_{6}(1,3,1 ; 1,1,2), B_{6}(1,3,1 ; 1,2,1), B_{6}(1,3,2 ; 1,1,1), B_{6}(1,4,1 ; 1,1,1)$.

(121) $B_{6}(2,1,1 ; 1,1,3), B_{6}(2,1,1 ; 1,2,2), B_{6}(2,1,1 ; 1,3,1), B_{6}(2,1,2 ; 1,1,2), B_{6}(2,1,2 ; 1,2,1)$.

(122) $B_{6}(2,1,2 ; 2,1,1), B_{6}(2,1,3 ; 1,1,1), B_{6}(2,2,1 ; 1,1,2), B_{6}(2,2,1 ; 1,2,1), B_{6}(2,2,1 ; 2,1,1)$.

(123) $B_{6}(2,2,2 ; 1,1,1), B_{6}(2,3,1 ; 1,1,1), B_{6}(3,1,1 ; 1,1,2), B_{6}(3,1,1 ; 1,2,1), B_{6}(3,1,1 ; 2,1,1)$.

(124) $B_{6}(3,1,2 ; 1,1,1), B_{6}(3,2,1 ; 1,1,1), B_{6}(4,1,1 ; 1,1,1)$.

(125) $B_{6}(1,1,3 ; 1,1,3), B_{6}(1,1,4 ; 1,1,2), B_{6}(1,1,5 ; 1,1,1), B_{6}(1,2,1 ; 1,1,4), B_{6}(1,2,2 ; 1,1,3)$.

(126) $B_{6}(1,2,3 ; 1,1,2), B_{6}(1,2,3 ; 1,2,1), B_{6}(1,2,4 ; 1,1,1), B_{6}(1,3,1 ; 1,1,3), B_{6}(1,3,1 ; 1,2,2)$.

(127) $B_{6}(1,3,1 ; 1,3,1), B_{6}(1,3,2 ; 1,1,2), B_{6}(1,3,2 ; 1,2,1), B_{6}(1,3,3 ; 1,1,1), B_{6}(1,4,1 ; 1,1,2)$.

(128) $B_{6}(1,4,1 ; 1,2,1), B_{6}(1,4,2 ; 1,1,1), B_{6}(1,5,1 ; 1,1,1), B_{6}(2,1,1 ; 1,1,4), B_{6}(2,1,1 ; 1,2,3)$.

(129) $B_{6}(2,1,1 ; 1,3,2), B_{6}(2,1,1 ; 1,4,1), B_{6}(2,1,2 ; 1,1,3), B_{6}(2,1,2 ; 1,2,2), B_{6}(2,1,2 ; 1,3,1)$.

(130) $B_{6}(2,1,2 ; 2,1,2), B_{6}(2,1,3 ; 1,1,2), B_{6}(2,1,3 ; 1,2,1), B_{6}(2,1,3 ; 2,1,1), B_{6}(2,1,4 ; 1,1,1)$.

(131) $B_{6}(2,2,1 ; 1,1,3), B_{6}(2,2,1 ; 1,3,1), B_{6}(2,2,1 ; 2,1,2), B_{6}(2,2,1 ; 2,2,1), B_{6}(2,2,2 ; 1,1,2)$.

(132) $B_{6}(2,2,2 ; 1,2,1), B_{6}(2,2,2 ; 2,1,1), B_{6}(2,2,3 ; 1,1,1), B_{6}(2,3,1 ; 1,1,2), B_{6}(2,3,1 ; 1,2,1)$.

(133) $B_{6}(2,3,1 ; 2,1,1), B_{6}(2,3,2 ; 1,1,1), B_{6}(2,4,1 ; 1,1,1), B_{6}(3,1,1 ; 1,1,3), B_{6}(3,1,1 ; 1,2,2)$.

(134) $B_{6}(3,1,1 ; 1,3,1), B_{6}(3,1,1 ; 2,1,2), B_{6}(3,1,1 ; 2,2,1), B_{6}(3,1,1 ; 3,1,1), B_{6}(3,1,2 ; 1,1,2)$.

(135) $B_{6}(3,1,2 ; 1,2,1), B_{6}(3,1,2 ; 2,1,1), B_{6}(3,1,3 ; 1,1,1), B_{6}(3,2,1 ; 1,1,2), B_{6}(3,2,1 ; 1,2,1)$.

(136) $B_{6}(3,2,1 ; 2,1,1), B_{6}(3,2,2 ; 1,1,1), B_{6}(3,3,1 ; 1,1,1), B_{6}(4,1,1 ; 1,1,2), B_{6}(4,1,1 ; 1,2,1)$.

(137) $B_{6}(4,1,1 ; 2,1,1), B_{6}(4,1,2 ; 1,1,1), B_{6}(4,2,1 ; 1,1,1), B_{6}(5,1,1 ; 1,1,1)$.

(138) $B_{6}(1,1,4 ; 1,1,3), B_{6}(1,1,5 ; 1,1,2), B_{6}(1,1,6 ; 1,1,1), B_{6}(1,2,1 ; 1,1,5), B_{6}(1,2,2 ; 1,1,4)$.

(139) $B_{6}(1,2,3 ; 1,1,3), B_{6}(1,2,4 ; 1,1,2), B_{6}(1,2,4 ; 1,2,1), B_{6}(1,2,5 ; 1,1,1), B_{6}(1,3,1 ; 1,1,4)$.

(140) $B_{6}(1,3,1 ; 1,2,3), B_{6}(1,3,2 ; 1,1,3), B_{6}(1,3,2 ; 1,3,1), B_{6}(1,3,4 ; 1,1,1), B_{6}(1,4,1 ; 1,1,3)$.

(141) $B_{6}(1,4,1 ; 1,2,2), B_{6}(1,4,1 ; 1,3,1), B_{6}(1,4,2 ; 1,1,2), B_{6}(1,4,2 ; 1,2,1), B_{6}(1,4,3 ; 1,1,1)$.

(142) $B_{6}(1,5,1 ; 1,1,2), B_{6}(1,5,1 ; 1,2,1), B_{6}(1,5,2 ; 1,1,1), B_{6}(1,6,1 ; 1,1,1), B_{6}(2,1,1 ; 1,1,5)$.

(143) $B_{6}(2,1,1 ; 1,2,4), B_{6}(2,1,1 ; 1,4,2), B_{6}(2,1,1 ; 1,5,1), B_{6}(2,1,2 ; 1,1,4), B_{6}(2,1,2 ; 1,2,3)$.

(144) $B_{6}(2,1,2 ; 1,3,2), B_{6}(2,1,2 ; 1,4,1), B_{6}(2,1,3 ; 1,1,3), B_{6}(2,1,3 ; 1,2,2), B_{6}(2,1,3 ; 1,3,1)$. 
(145) $B_{6}(2,1,3 ; 2,1,2), B_{6}(2,1,4 ; 1,1,2), B_{6}(2,1,4 ; 1,2,1), B_{6}(2,1,4 ; 2,1,1), B_{6}(2,1,5 ; 1,1,1)$.

(146) $B_{6}(2,2,1 ; 1,1,4), B_{6}(2,2,1 ; 1,4,1), B_{6}(2,2,1 ; 2,1,3), B_{6}(2,2,2 ; 1,1,3), B_{6}(2,2,2 ; 1,3,1)$.

(147) $B_{6}(2,2,2 ; 2,1,2), B_{6}(2,2,3 ; 1,1,2), B_{6}(2,2,3 ; 1,2,1), B_{6}(2,2,3 ; 2,1,1), B_{6}(2,2,4 ; 1,1,1)$.

(148) $B_{6}(2,3,1 ; 1,1,3), B_{6}(2,3,1 ; 1,3,1), B_{6}(2,3,1 ; 2,1,2), B_{6}(2,3,1 ; 2,2,1), B_{6}(2,3,2 ; 2,1,1)$.

(149) $B_{6}(2,3,3 ; 1,1,1), B_{6}(2,4,1 ; 1,1,2), B_{6}(2,4,1 ; 1,2,1), B_{6}(2,4,1 ; 2,1,1), B_{6}(2,4,2 ; 1,1,1)$.

(150) $B_{6}(2,5,1 ; 1,1,1), B_{6}(3,1,1 ; 1,1,4), B_{6}(3,1,1 ; 1,2,3), B_{6}(3,1,1 ; 1,3,2), B_{6}(3,1,1 ; 1,4,1)$.

(151) $B_{6}(3,1,1 ; 2,1,3), B_{6}(3,1,1 ; 2,2,2), B_{6}(3,1,1 ; 2,3,1), B_{6}(3,1,2 ; 1,1,3), B_{6}(3,1,2 ; 1,2,2)$.

(152) $B_{6}(3,1,2 ; 1,3,1), B_{6}(3,1,2 ; 2,1,2), B_{6}(3,1,2 ; 2,2,1), B_{6}(3,1,3 ; 1,1,2), B_{6}(3,1,3 ; 1,2,1)$.

(153) $B_{6}(3,1,3 ; 2,1,1), B_{6}(3,1,4 ; 1,1,1), B_{6}(3,2,1 ; 1,1,3), B_{6}(3,2,1 ; 1,3,1), B_{6}(3,2,1 ; 2,2,1)$.

(154) $B_{6}(3,2,1 ; 3,1,1), B_{6}(3,2,2 ; 1,1,2), B_{6}(3,2,2 ; 1,2,1), B_{6}(3,2,2 ; 2,1,1), B_{6}(3,2,3 ; 1,1,1)$.

(155) $B_{6}(3,3,1 ; 2,1,1), B_{6}(3,3,2 ; 1,1,1), B_{6}(3,4,1 ; 1,1,1), B_{6}(4,1,1 ; 1,1,3), B_{6}(4,1,1 ; 1,2,2)$.

(156) $B_{6}(4,1,1 ; 1,3,1), B_{6}(4,1,1 ; 2,1,2), B_{6}(4,1,1 ; 2,2,1), B_{6}(4,1,1 ; 3,1,1), B_{6}(4,1,2 ; 1,1,2)$.

(157) $B_{6}(4,1,2 ; 1,2,1), B_{6}(4,1,2 ; 2,1,1), B_{6}(4,1,3 ; 1,1,1), B_{6}(4,2,1 ; 1,1,2), B_{6}(4,2,1 ; 1,2,1)$.

(158) $B_{6}(4,2,1 ; 2,1,1), B_{6}(4,2,2 ; 1,1,1), B_{6}(4,3,1 ; 1,1,1), B_{6}(5,1,1 ; 1,1,2), B_{6}(5,1,1 ; 1,2,1)$.

(159) $B_{6}(5,1,1 ; 2,1,1), B_{6}(5,1,2 ; 1,1,1), B_{6}(5,2,1 ; 1,1,1), B_{6}(6,1,1 ; 1,1,1)$.

(160) $B_{6}(1,1,4 ; 1,1,4), B_{6}(1,1,5 ; 1,1,3), B_{6}(1,1,6 ; 1,1,2), B_{6}(1,1,7 ; 1,1,1), B_{6}(1,2,1 ; 1,1,6)$.

(161) $B_{6}(1,2,2 ; 1,1,5), B_{6}(1,2,3 ; 1,1,4), B_{6}(1,2,5 ; 1,1,2), B_{6}(1,2,5 ; 1,2,1), B_{6}(1,2,6 ; 1,1,1)$.

(162) $B_{6}(1,3,1 ; 1,1,5), B_{6}(1,3,2 ; 1,1,4), B_{6}(1,3,5 ; 1,1,1), B_{6}(1,4,1 ; 1,1,4), B_{6}(1,4,1 ; 1,2,3)$.

(163) $B_{6}(1,4,1 ; 1,3,2), B_{6}(1,4,1 ; 1,4,1), B_{6}(1,5,1 ; 1,1,3), B_{6}(1,5,1 ; 1,2,2), B_{6}(1,5,1 ; 1,3,1)$.

(164) $B_{6}(1,5,2 ; 1,1,2), B_{6}(1,5,2 ; 1,2,1), B_{6}(1,5,3 ; 1,1,1), B_{6}(1,6,1 ; 1,1,2), B_{6}(1,6,1 ; 1,2,1)$.

(165) $B_{6}(1,6,2 ; 1,1,1), B_{6}(1,7,1 ; 1,1,1), B_{6}(2,1,1 ; 1,1,6), B_{6}(2,1,1 ; 1,2,5), B_{6}(2,1,1 ; 1,5,2)$.

(166) $B_{6}(2,1,1 ; 1,6,1), B_{6}(2,1,2 ; 1,1,5), B_{6}(2,1,2 ; 1,5,1), B_{6}(2,1,3 ; 1,1,4), B_{6}(2,1,3 ; 1,2,3)$.

(167) $B_{6}(2,1,3 ; 1,3,2), B_{6}(2,1,3 ; 1,4,1), B_{6}(2,1,3 ; 2,1,3), B_{6}(2,1,4 ; 1,1,3), B_{6}(2,1,4 ; 1,2,2)$.

(168) $B_{6}(2,1,4 ; 1,3,1), B_{6}(2,1,4 ; 2,1,2), B_{6}(2,1,5 ; 1,1,2), B_{6}(2,1,5 ; 1,2,1), B_{6}(2,1,5 ; 2,1,1)$.

(169) $B_{6}(2,1,6 ; 1,1,1), B_{6}(2,2,1 ; 1,1,5), B_{6}(2,2,1 ; 1,5,1), B_{6}(2,2,1 ; 2,1,4), B_{6}(2,2,2 ; 1,1,4)$.

(170) $B_{6}(2,2,2 ; 1,4,1), B_{6}(2,2,2 ; 2,1,3), B_{6}(2,2,3 ; 2,1,2), B_{6}(2,2,4 ; 1,1,2), B_{6}(2,2,4 ; 1,2,1)$.

(171) $B_{6}(2,2,4 ; 2,1,1), B_{6}(2,2,5 ; 1,1,1), B_{6}(2,3,1 ; 1,1,4), B_{6}(2,3,1 ; 1,4,1), B_{6}(2,3,4 ; 1,1,1)$.

(172) $B_{6}(2,4,1 ; 2,1,2), B_{6}(2,4,1 ; 2,2,1), B_{6}(2,4,2 ; 2,1,1), B_{6}(2,5,1 ; 1,1,2), B_{6}(2,5,1 ; 1,2,1)$.

(173) $B_{6}(2,5,1 ; 2,1,1), B_{6}(2,5,2 ; 1,1,1), B_{6}(2,6,1 ; 1,1,1), B_{6}(3,1,1 ; 1,1,5), B_{6}(3,1,1 ; 1,5,1)$.

(174) $B_{6}(3,1,1 ; 2,1,4), B_{6}(3,1,1 ; 2,3,2), B_{6}(3,1,1 ; 2,4,1), B_{6}(3,1,2 ; 1,1,4), B_{6}(3,1,2 ; 1,2,3)$.

(175) $B_{6}(3,1,2 ; 1,3,2), B_{6}(3,1,2 ; 1,4,1), B_{6}(3,1,2 ; 2,1,3), B_{6}(3,1,2 ; 2,2,2), B_{6}(3,1,2 ; 2,3,1)$.

(176) $B_{6}(3,1,3 ; 1,1,3), B_{6}(3,1,3 ; 1,2,2), B_{6}(3,1,3 ; 1,3,1), B_{6}(3,1,3 ; 2,1,2), B_{6}(3,1,4 ; 1,1,2)$.

(177) $B_{6}(3,1,4 ; 1,2,1), B_{6}(3,1,4 ; 2,1,1), B_{6}(3,1,5 ; 1,1,1), B_{6}(3,2,1 ; 1,1,4), B_{6}(3,2,1 ; 1,4,1)$.

(178) $B_{6}(3,2,1 ; 2,3,1), B_{6}(3,2,1 ; 3,2,1), B_{6}(3,2,3 ; 1,1,2), B_{6}(3,2,3 ; 1,2,1), B_{6}(3,2,3 ; 2,1,1)$.

(179) $B_{6}(3,2,4 ; 1,1,1), B_{6}(3,3,1 ; 3,1,1), B_{6}(3,3,2 ; 2,1,1), B_{6}(3,3,3 ; 1,1,1), B_{6}(3,4,1 ; 2,1,1)$.

(180) $B_{6}(3,5,1 ; 1,1,1), B_{6}(4,1,1 ; 1,1,4), B_{6}(4,1,1 ; 1,2,3), B_{6}(4,1,1 ; 1,3,2), B_{6}(4,1,1 ; 1,4,1)$. 
(181) $B_{6}(4,1,1 ; 2,2,2), B_{6}(4,1,1 ; 2,3,1), B_{6}(4,1,1 ; 3,2,1), B_{6}(4,1,2 ; 1,1,3), B_{6}(4,1,2 ; 1,2,2)$.

(182) $B_{6}(4,1,2 ; 1,3,1), B_{6}(4,1,2 ; 2,2,1), B_{6}(4,1,3 ; 1,1,2), B_{6}(4,1,3 ; 1,2,1), B_{6}(4,1,4 ; 1,1,1)$.

(183) $B_{6}(4,2,1 ; 2,2,1), B_{6}(4,2,2 ; 1,1,2), B_{6}(4,2,2 ; 1,2,1), B_{6}(4,2,2 ; 2,1,1), B_{6}(4,2,3 ; 1,1,1)$.

(184) $B_{6}(4,3,2 ; 1,1,1), B_{6}(5,1,1 ; 1,1,3), B_{6}(5,1,1 ; 1,2,2), B_{6}(5,1,1 ; 1,3,1), B_{6}(5,1,1 ; 2,1,2)$.

(185) $B_{6}(5,1,1 ; 2,2,1), B_{6}(5,1,1 ; 3,1,1), B_{6}(5,1,2 ; 1,1,2), B_{6}(5,1,2 ; 1,2,1), B_{6}(5,1,2 ; 2,1,1)$.

(186) $B_{6}(5,1,3 ; 1,1,1), B_{6}(5,2,1 ; 1,1,2), B_{6}(5,2,1 ; 1,2,1), B_{6}(5,2,1 ; 2,1,1), B_{6}(5,2,2 ; 1,1,1)$.

(187) $B_{6}(5,3,1 ; 1,1,1), B_{6}(6,1,1 ; 1,1,2), B_{6}(6,1,1 ; 1,2,1), B_{6}(6,1,1 ; 2,1,1), B_{6}(6,1,2 ; 1,1,1)$.

(188) $B_{6}(6,2,1 ; 1,1,1), B_{6}(7,1,1 ; 1,1,1)$.

(189) $B_{7}(1,1,1 ; 1,1,1 ; 1)$.

(190) $B_{7}(1,1,1 ; 1,1,1 ; 2), B_{7}(1,1,2 ; 1,1,1 ; 1), B_{7}(1,2,1 ; 1,1,1 ; 1), B_{7}(2,1,1 ; 1,1,1 ; 1)$.

(191) $B_{7}(1,1,1 ; 1,1,1 ; 3), B_{7}(1,1,2 ; 1,1,1 ; 2), B_{7}(1,1,2 ; 1,1,2 ; 1), B_{7}(1,1,3 ; 1,1,1 ; 1)$.

(192) $B_{7}(1,2,1 ; 1,1,1 ; 2), B_{7}(1,2,1 ; 1,1,2 ; 1), B_{7}(1,2,1 ; 1,2,1 ; 1), B_{7}(1,2,2 ; 1,1,1 ; 1)$.

(193) $B_{7}(1,3,1 ; 1,1,1 ; 1), B_{7}(2,1,1 ; 1,1,1 ; 2), B_{7}(2,1,1 ; 1,1,2 ; 1), B_{7}(2,1,1 ; 1,2,1 ; 1)$.

(194) $B_{7}(2,1,1 ; 2,1,1 ; 1), B_{7}(2,1,2 ; 1,1,1 ; 1), B_{7}(2,2,1 ; 1,1,1 ; 1), B_{7}(3,1,1 ; 1,1,1 ; 1)$.

(195) $B_{7}(1,1,1 ; 1,1,1 ; 4), B_{7}(1,1,2 ; 1,1,1 ; 3), B_{7}(1,1,2 ; 1,1,2 ; 2), B_{7}(1,1,3 ; 1,1,1 ; 2)$.

(196) $B_{7}(1,1,3 ; 1,1,2 ; 1), B_{7}(1,1,4 ; 1,1,1 ; 1), B_{7}(1,2,1 ; 1,1,1 ; 3), B_{7}(1,2,1 ; 1,1,2 ; 2)$.

(197) $B_{7}(1,2,1 ; 1,1,3 ; 1), B_{7}(1,2,1 ; 1,2,1 ; 2), B_{7}(1,2,2 ; 1,1,1 ; 2), B_{7}(1,2,2 ; 1,1,2 ; 1)$.

(198) $B_{7}(1,2,2 ; 1,2,1 ; 1), B_{7}(1,2,3 ; 1,1,1 ; 1), B_{7}(1,3,1 ; 1,1,1 ; 2), B_{7}(1,3,1 ; 1,1,2 ; 1)$.

(199) $B_{7}(1,3,1 ; 1,2,1 ; 1), B_{7}(1,3,2 ; 1,1,1 ; 1), B_{7}(1,4,1 ; 1,1,1 ; 1), B_{7}(2,1,1 ; 1,1,1 ; 3)$.

(200) $B_{7}(2,1,1 ; 1,1,2 ; 2), B_{7}(2,1,1 ; 1,1,3 ; 1), B_{7}(2,1,1 ; 1,2,1 ; 2), B_{7}(2,1,1 ; 1,2,2 ; 1)$.

(201) $B_{7}(2,1,1 ; 1,3,1 ; 1), B_{7}(2,1,1 ; 2,1,1 ; 2), B_{7}(2,1,2 ; 1,1,1 ; 2), B_{7}(2,1,2 ; 1,1,2 ; 1)$.

(202) $B_{7}(2,1,2 ; 1,2,1 ; 1), B_{7}(2,1,3 ; 1,1,1 ; 1), B_{7}(2,2,1 ; 1,1,1 ; 2), B_{7}(2,2,1 ; 1,2,1 ; 1)$.

(203) $B_{7}(2,2,1 ; 2,1,1 ; 1), B_{7}(2,2,2 ; 1,1,1 ; 1), B_{7}(2,3,1 ; 1,1,1 ; 1), B_{7}(3,1,1 ; 1,1,1 ; 2)$.

(204) $B_{7}(3,1,1 ; 1,1,2 ; 1), B_{7}(3,1,1 ; 1,2,1 ; 1), B_{7}(3,1,1 ; 2,1,1 ; 1), B_{7}(3,1,2 ; 1,1,1 ; 1)$.

(205) $B_{7}(3,2,1 ; 1,1,1 ; 1), B_{7}(4,1,1 ; 1,1,1 ; 1)$.

(206) $B_{7}(1,1,1 ; 1,1,1 ; 5), B_{7}(1,1,2 ; 1,1,1 ; 4), B_{7}(1,1,2 ; 1,1,2 ; 3), B_{7}(1,1,3 ; 1,1,1 ; 3)$.

(207) $B_{7}(1,1,3 ; 1,1,2 ; 2), B_{7}(1,1,3 ; 1,1,3 ; 1), B_{7}(1,1,4 ; 1,1,1 ; 2), B_{7}(1,1,4 ; 1,1,2 ; 1)$.

(208) $B_{7}(1,1,5 ; 1,1,1 ; 1), B_{7}(1,2,1 ; 1,1,1 ; 4), B_{7}(1,2,1 ; 1,1,2 ; 3), B_{7}(1,2,1 ; 1,1,4 ; 1)$.

(209) $B_{7}(1,2,1 ; 1,2,1 ; 3), B_{7}(1,2,2 ; 1,1,1 ; 3), B_{7}(1,2,2 ; 1,1,2 ; 2), B_{7}(1,2,2 ; 1,1,3 ; 1)$.

(210) $B_{7}(1,2,2 ; 1,2,1 ; 2), B_{7}(1,2,3 ; 1,1,1 ; 2), B_{7}(1,2,3 ; 1,1,2 ; 1), B_{7}(1,2,4 ; 1,1,1 ; 1)$.

(211) $B_{7}(1,3,1 ; 1,1,1 ; 3), B_{7}(1,3,1 ; 1,1,2 ; 2), B_{7}(1,3,1 ; 1,1,3 ; 1), B_{7}(1,3,1 ; 1,2,1 ; 2)$.

(212) $B_{7}(1,3,1 ; 1,2,2 ; 1), B_{7}(1,3,1 ; 1,3,1 ; 1), B_{7}(1,3,2 ; 1,1,1 ; 2), B_{7}(1,3,2 ; 1,1,2 ; 1)$.

(213) $B_{7}(1,3,2 ; 1,2,1 ; 1), B_{7}(1,3,3 ; 1,1,1 ; 1), B_{7}(1,4,1 ; 1,1,1 ; 2), B_{7}(1,4,1 ; 1,1,2 ; 1)$.

(214) $B_{7}(1,4,1 ; 1,2,1 ; 1), B_{7}(1,4,2 ; 1,1,1 ; 1), B_{7}(1,5,1 ; 1,1,1 ; 1), B_{7}(2,1,1 ; 1,1,1 ; 4)$.

(215) $B_{7}(2,1,1 ; 1,1,2 ; 3), B_{7}(2,1,1 ; 1,1,4 ; 1), B_{7}(2,1,1 ; 1,2,1 ; 3), B_{7}(2,1,1 ; 1,2,2 ; 2)$.

(216) $B_{7}(2,1,1 ; 1,3,1 ; 2), B_{7}(2,1,1 ; 1,3,2 ; 1), B_{7}(2,1,1 ; 1,4,1 ; 1), B_{7}(2,1,1 ; 2,1,1 ; 3)$. 
(217) $B_{7}(2,1,2 ; 1,1,1 ; 3), B_{7}(2,1,2 ; 1,1,3 ; 1), B_{7}(2,1,2 ; 1,2,1 ; 2), B_{7}(2,1,2 ; 1,2,2 ; 1)$.

(218) $B_{7}(2,1,2 ; 1,3,1 ; 1), B_{7}(2,1,3 ; 1,1,2 ; 1), B_{7}(2,1,3 ; 1,2,1 ; 1), B_{7}(2,1,4 ; 1,1,1 ; 1)$. (219) $B_{7}(2,2,1 ; 1,1,1 ; 3), B_{7}(2,2,1 ; 1,2,1 ; 2), B_{7}(2,2,1 ; 1,3,1 ; 1), B_{7}(2,2,1 ; 2,1,1 ; 2)$. (220) $B_{7}(2,2,1 ; 2,2,1 ; 1), B_{7}(2,2,2 ; 1,1,1 ; 2), B_{7}(2,2,2 ; 1,2,1 ; 1), B_{7}(2,2,3 ; 1,1,1 ; 1)$. (221) $B_{7}(2,3,1 ; 1,1,1 ; 2), B_{7}(2,3,1 ; 1,2,1 ; 1), B_{7}(2,3,1 ; 2,1,1 ; 1), B_{7}(2,3,2 ; 1,1,1 ; 1)$. (222) $B_{7}(2,4,1 ; 1,1,1 ; 1), B_{7}(3,1,1 ; 1,1,1 ; 3), B_{7}(3,1,1 ; 1,1,2 ; 2), B_{7}(3,1,1 ; 1,1,3 ; 1)$. (223) $B_{7}(3,1,1 ; 1,2,1 ; 2), B_{7}(3,1,1 ; 1,2,2 ; 1), B_{7}(3,1,1 ; 1,3,1 ; 1), B_{7}(3,1,1 ; 2,1,1 ; 2)$. (224) $B_{7}(3,1,1 ; 2,2,1 ; 1), B_{7}(3,1,2 ; 1,1,1 ; 2), B_{7}(3,1,2 ; 1,1,2 ; 1), B_{7}(3,1,2 ; 1,2,1 ; 1)$. (225) $B_{7}(3,1,3 ; 1,1,1 ; 1), B_{7}(3,2,1 ; 1,1,1 ; 2), B_{7}(3,2,1 ; 1,2,1 ; 1), B_{7}(3,2,2 ; 1,1,1 ; 1)$. (226) $B_{7}(4,1,1 ; 1,1,1 ; 2), B_{7}(4,1,1 ; 1,1,2 ; 1), B_{7}(4,1,1 ; 1,2,1 ; 1), B_{7}(4,1,1 ; 2,1,1 ; 1)$. (227) $B_{7}(4,1,2 ; 1,1,1 ; 1), B_{7}(4,2,1 ; 1,1,1 ; 1), B_{7}(5,1,1 ; 1,1,1 ; 1)$.

(228) $B_{7}(1,1,1 ; 1,1,1 ; 6), B_{7}(1,1,2 ; 1,1,1 ; 5), B_{7}(1,1,2 ; 1,1,2 ; 4), B_{7}(1,1,3 ; 1,1,1 ; 4)$. (229) $B_{7}(1,1,3 ; 1,1,3 ; 2), B_{7}(1,1,4 ; 1,1,2 ; 2), B_{7}(1,1,4 ; 1,1,3 ; 1), B_{7}(1,1,5 ; 1,1,1 ; 2)$. (230) $B_{7}(1,1,5 ; 1,1,2 ; 1), B_{7}(1,1,6 ; 1,1,1 ; 1), B_{7}(1,2,1 ; 1,1,1 ; 5), B_{7}(1,2,1 ; 1,1,2 ; 4)$. (231) $B_{7}(1,2,1 ; 1,1,5 ; 1), B_{7}(1,2,1 ; 1,2,1 ; 4), B_{7}(1,2,2 ; 1,1,1 ; 4), B_{7}(1,2,2 ; 1,1,2 ; 3)$. (232) $B_{7}(1,2,2 ; 1,1,4 ; 1), B_{7}(1,2,2 ; 1,2,1 ; 3), B_{7}(1,2,3 ; 1,1,1 ; 3), B_{7}(1,2,3 ; 1,1,2 ; 2)$. (233) $B_{7}(1,2,3 ; 1,1,3 ; 1), B_{7}(1,2,4 ; 1,1,2 ; 1), B_{7}(1,2,5 ; 1,1,1 ; 1), B_{7}(1,3,1 ; 1,1,1 ; 4)$. (234) $B_{7}(1,3,1 ; 1,2,1 ; 3), B_{7}(1,3,1 ; 1,3,1 ; 2), B_{7}(1,3,2 ; 1,1,1 ; 3), B_{7}(1,3,2 ; 1,1,2 ; 2)$. (235) $B_{7}(1,3,2 ; 1,2,1 ; 2), B_{7}(1,3,3 ; 1,1,1 ; 2), B_{7}(1,4,1 ; 1,1,1 ; 3), B_{7}(1,4,1 ; 1,1,2 ; 2)$. (236) $B_{7}(1,4,1 ; 1,1,3 ; 1), B_{7}(1,4,1 ; 1,2,1 ; 2), B_{7}(1,4,1 ; 1,2,2 ; 1), B_{7}(1,4,1 ; 1,3,1 ; 1)$. (237) $B_{7}(1,4,2 ; 1,1,1 ; 2), B_{7}(1,4,2 ; 1,1,2 ; 1), B_{7}(1,4,2 ; 1,2,1 ; 1), B_{7}(1,4,3 ; 1,1,1 ; 1)$. (238) $B_{7}(1,5,1 ; 1,1,1 ; 2), B_{7}(1,5,1 ; 1,1,2 ; 1), B_{7}(1,5,1 ; 1,2,1 ; 1), B_{7}(1,5,2 ; 1,1,1 ; 1)$. (239) $B_{7}(1,6,1 ; 1,1,1 ; 1), B_{7}(2,1,1 ; 1,1,1 ; 5), B_{7}(2,1,1 ; 1,1,2 ; 4), B_{7}(2,1,1 ; 1,1,5 ; 1)$. (240) $B_{7}(2,1,1 ; 1,2,1 ; 4), B_{7}(2,1,1 ; 1,2,2 ; 3), B_{7}(2,1,1 ; 1,3,1 ; 3), B_{7}(2,1,1 ; 1,3,2 ; 2)$. (241) $B_{7}(2,1,1 ; 1,4,1 ; 2), B_{7}(2,1,1 ; 1,4,2 ; 1), B_{7}(2,1,1 ; 1,5,1 ; 1), B_{7}(2,1,1 ; 2,1,1 ; 4)$. (242) $B_{7}(2,1,2 ; 1,1,1 ; 4), B_{7}(2,1,2 ; 1,1,4 ; 1), B_{7}(2,1,2 ; 1,3,1 ; 2), B_{7}(2,1,2 ; 1,3,2 ; 1)$. (243) $B_{7}(2,1,2 ; 1,4,1 ; 1), B_{7}(2,1,3 ; 1,1,3 ; 1), B_{7}(2,1,3 ; 1,2,2 ; 1), B_{7}(2,1,3 ; 1,3,1 ; 1)$. (244) $B_{7}(2,1,4 ; 1,1,2 ; 1), B_{7}(2,1,5 ; 1,1,1 ; 1), B_{7}(2,2,1 ; 1,1,1 ; 4), B_{7}(2,2,1 ; 1,2,1 ; 3)$. (245) $B_{7}(2,2,1 ; 1,3,1 ; 2), B_{7}(2,2,1 ; 1,4,1 ; 1), B_{7}(2,2,1 ; 2,1,1 ; 3), B_{7}(2,2,1 ; 2,2,1 ; 2)$. (246) $B_{7}(2,2,2 ; 1,1,1 ; 3), B_{7}(2,2,2 ; 1,2,1 ; 2), B_{7}(2,2,2 ; 1,3,1 ; 1), B_{7}(2,2,4 ; 1,1,1 ; 1)$. (247) $B_{7}(2,3,1 ; 1,1,1 ; 3), B_{7}(2,3,1 ; 1,2,1 ; 2), B_{7}(2,3,1 ; 1,3,1 ; 1), B_{7}(2,3,1 ; 2,2,1 ; 1)$. (248) $B_{7}(2,3,2 ; 1,1,1 ; 2), B_{7}(2,3,3 ; 1,1,1 ; 1), B_{7}(2,4,1 ; 2,1,1 ; 1), B_{7}(2,4,2 ; 1,1,1 ; 1)$. (249) $B_{7}(2,5,1 ; 1,1,1 ; 1), B_{7}(3,1,1 ; 1,1,1 ; 4), B_{7}(3,1,1 ; 1,2,1 ; 3), B_{7}(3,1,1 ; 1,3,1 ; 2)$. (250) $B_{7}(3,1,1 ; 1,4,1 ; 1), B_{7}(3,1,1 ; 2,1,1 ; 3), B_{7}(3,1,1 ; 2,3,1 ; 1), B_{7}(3,1,2 ; 1,2,1 ; 2)$. (251) $B_{7}(3,1,2 ; 1,2,2 ; 1), B_{7}(3,1,2 ; 1,3,1 ; 1), B_{7}(3,1,3 ; 1,2,1 ; 1), B_{7}(3,2,1 ; 1,1,1 ; 3)$. (252) $B_{7}(3,2,1 ; 1,2,1 ; 2), B_{7}(3,2,1 ; 1,3,1 ; 1), B_{7}(3,2,2 ; 1,2,1 ; 1), B_{7}(3,2,3 ; 1,1,1 ; 1)$. 
(253) $B_{7}(4,1,1 ; 1,1,1 ; 3), B_{7}(4,1,1 ; 1,1,2 ; 2), B_{7}(4,1,1 ; 1,1,3 ; 1), B_{7}(4,1,1 ; 1,2,1 ; 2)$.

(254) $B_{7}(4,1,1 ; 1,2,2 ; 1), B_{7}(4,1,1 ; 1,3,1 ; 1), B_{7}(4,1,1 ; 2,2,1 ; 1), B_{7}(4,1,2 ; 1,1,1 ; 2)$.

(255) $B_{7}(4,1,2 ; 1,1,2 ; 1), B_{7}(4,1,2 ; 1,2,1 ; 1), B_{7}(4,1,3 ; 1,1,1 ; 1), B_{7}(4,2,2 ; 1,1,1 ; 1)$.

(256) $B_{7}(5,1,1 ; 1,1,1 ; 2), B_{7}(5,1,1 ; 1,1,2 ; 1), B_{7}(5,1,1 ; 1,2,1 ; 1), B_{7}(5,1,1 ; 2,1,1 ; 1)$.

(257) $B_{7}(5,1,2 ; 1,1,1 ; 1), B_{7}(5,2,1 ; 1,1,1 ; 1), B_{7}(6,1,1 ; 1,1,1 ; 1)$.

(258) $B_{8}(1,1,1,1 ; 1,1,1,1)$.

(259) $B_{8}(1,1,1,2 ; 1,1,1,1), B_{8}(1,1,2,1 ; 1,1,1,1), B_{8}(1,2,1,1 ; 1,1,1,1), B_{8}(2,1,1,1 ; 1,1,1,1)$.

(260) $B_{8}(1,1,1,2 ; 1,1,1,2), B_{8}(1,1,1,3 ; 1,1,1,1), B_{8}(1,1,2,1 ; 1,1,1,2), B_{8}(1,1,2,1 ; 1,1,2,1)$.

(261) $B_{8}(1,1,2,2 ; 1,1,1,1), B_{8}(1,1,3,1 ; 1,1,1,1), B_{8}(1,2,1,1 ; 1,1,1,2), B_{8}(1,2,1,1 ; 1,1,2,1)$.

(262) $B_{8}(1,2,1,1 ; 1,2,1,1), B_{8}(1,2,1,2 ; 1,1,1,1), B_{8}(1,2,2,1 ; 1,1,1,1), B_{8}(1,3,1,1 ; 1,1,1,1)$.

(263) $B_{8}(2,1,1,1 ; 1,1,1,2), B_{8}(2,1,1,1 ; 1,1,2,1), B_{8}(2,1,1,1 ; 1,2,1,1), B_{8}(2,1,1,1 ; 2,1,1,1)$.

(264) $B_{8}(2,1,1,2 ; 1,1,1,1), B_{8}(2,1,2,1 ; 1,1,1,1), B_{8}(2,2,1,1 ; 1,1,1,1), B_{8}(3,1,1,1 ; 1,1,1,1)$.

(265) $B_{8}(1,1,1,3 ; 1,1,1,2), B_{8}(1,1,1,4 ; 1,1,1,1), B_{8}(1,1,2,1 ; 1,1,1,3), B_{8}(1,1,2,2 ; 1,1,1,2)$.

(266) $B_{8}(1,1,2,2 ; 1,1,2,1), B_{8}(1,1,2,3 ; 1,1,1,1), B_{8}(1,1,3,1 ; 1,1,1,2), B_{8}(1,1,3,1 ; 1,1,2,1)$.

(267) $B_{8}(1,1,3,2 ; 1,1,1,1), B_{8}(1,1,4,1 ; 1,1,1,1), B_{8}(1,2,1,1 ; 1,1,1,3), B_{8}(1,2,1,1 ; 1,1,3,1)$.

(268) $B_{8}(1,2,1,2 ; 1,1,2,1), B_{8}(1,2,1,2 ; 1,2,1,1), B_{8}(1,2,1,3 ; 1,1,1,1), B_{8}(1,2,2,1 ; 1,1,1,2)$.

(269) $B_{8}(1,2,2,1 ; 1,2,1,1), B_{8}(1,2,2,2 ; 1,1,1,1), B_{8}(1,2,3,1 ; 1,1,1,1), B_{8}(1,3,1,1 ; 1,1,1,2)$.

(270) $B_{8}(1,3,1,1 ; 1,1,2,1), B_{8}(1,3,1,1 ; 1,2,1,1), B_{8}(1,3,1,2 ; 1,1,1,1), B_{8}(1,3,2,1 ; 1,1,1,1)$.

(271) $B_{8}(1,4,1,1 ; 1,1,1,1), B_{8}(2,1,1,1 ; 1,1,1,3), B_{8}(2,1,1,1 ; 1,1,2,2), B_{8}(2,1,1,1 ; 1,1,3,1)$.

(272) $B_{8}(2,1,1,1 ; 1,2,2,1), B_{8}(2,1,1,1 ; 1,3,1,1), B_{8}(2,1,1,2 ; 1,1,1,2), B_{8}(2,1,1,2 ; 1,1,2,1)$.

(273) $B_{8}(2,1,1,2 ; 1,2,1,1), B_{8}(2,1,1,2 ; 2,1,1,1), B_{8}(2,1,1,3 ; 1,1,1,1), B_{8}(2,1,2,1 ; 1,1,1,2)$.

(274) $B_{8}(2,1,2,1 ; 1,1,2,1), B_{8}(2,1,2,1 ; 2,1,1,1), B_{8}(2,1,2,2 ; 1,1,1,1), B_{8}(2,1,3,1 ; 1,1,1,1)$.

(275) $B_{8}(2,2,1,1 ; 1,1,2,1), B_{8}(2,2,1,1 ; 1,2,1,1), B_{8}(2,2,1,1 ; 2,1,1,1), B_{8}(2,2,1,2 ; 1,1,1,1)$.

(276) $B_{8}(2,2,2,1 ; 1,1,1,1), B_{8}(2,3,1,1 ; 1,1,1,1), B_{8}(3,1,1,1 ; 1,1,1,2), B_{8}(3,1,1,1 ; 1,1,2,1)$.

(277) $B_{8}(3,1,1,1 ; 1,2,1,1), B_{8}(3,1,1,1 ; 2,1,1,1), B_{8}(3,1,1,2 ; 1,1,1,1), B_{8}(3,1,2,1 ; 1,1,1,1)$.

(278) $B_{8}(3,2,1,1 ; 1,1,1,1), B_{8}(4,1,1,1 ; 1,1,1,1)$.

(279) $B_{8}(1,1,1,4 ; 1,1,1,2), B_{8}(1,1,1,5 ; 1,1,1,1), B_{8}(1,1,2,1 ; 1,1,1,4), B_{8}(1,1,2,2 ; 1,1,1,3)$.

(280) $B_{8}(1,1,2,2 ; 1,1,2,2), B_{8}(1,1,2,3 ; 1,1,2,1), B_{8}(1,1,2,4 ; 1,1,1,1), B_{8}(1,1,3,1 ; 1,1,1,3)$.

(281) $B_{8}(1,1,3,1 ; 1,1,2,2), B_{8}(1,1,3,2 ; 1,1,1,2), B_{8}(1,1,4,1 ; 1,1,1,2), B_{8}(1,1,4,1 ; 1,1,2,1)$.

(282) $B_{8}(1,1,4,2 ; 1,1,1,1), B_{8}(1,1,5,1 ; 1,1,1,1), B_{8}(1,2,1,1 ; 1,1,1,4), B_{8}(1,2,1,1 ; 1,1,4,1)$.

(283) $B_{8}(1,2,1,2 ; 1,1,3,1), B_{8}(1,2,1,3 ; 1,1,2,1), B_{8}(1,2,1,4 ; 1,1,1,1), B_{8}(1,2,2,1 ; 1,1,1,3)$.

(284) $B_{8}(1,2,2,1 ; 1,2,1,2), B_{8}(1,2,2,3 ; 1,1,1,1), B_{8}(1,2,3,1 ; 1,1,1,2), B_{8}(1,2,3,2 ; 1,1,1,1)$.

(285) $B_{8}(1,2,4,1 ; 1,1,1,1), B_{8}(1,3,1,1 ; 1,2,1,2), B_{8}(1,3,1,1 ; 1,2,2,1), B_{8}(1,3,1,1 ; 1,3,1,1)$.

(286) $B_{8}(1,3,1,2 ; 1,2,1,1), B_{8}(1,3,2,1 ; 1,2,1,1), B_{8}(1,3,2,2 ; 1,1,1,1), B_{8}(1,4,1,1 ; 1,1,1,2)$.

(287) $B_{8}(1,4,1,1 ; 1,1,2,1), B_{8}(1,4,1,1 ; 1,2,1,1), B_{8}(1,4,1,2 ; 1,1,1,1), B_{8}(1,4,2,1 ; 1,1,1,1)$.

(288) $B_{8}(1,5,1,1 ; 1,1,1,1), B_{8}(2,1,1,1 ; 1,1,1,4), B_{8}(2,1,1,1 ; 1,1,3,2), B_{8}(2,1,1,1 ; 1,1,4,1)$. 
(289) $B_{8}(2,1,1,1 ; 1,2,3,1), B_{8}(2,1,1,1 ; 1,4,1,1), B_{8}(2,1,1,2 ; 1,1,2,2), B_{8}(2,1,1,2 ; 1,1,3,1)$.

(290) $B_{8}(2,1,1,2 ; 1,2,2,1), B_{8}(2,1,1,2 ; 2,1,1,2), B_{8}(2,1,1,3 ; 1,1,1,2), B_{8}(2,1,1,3 ; 1,1,2,1)$.

(291) $B_{8}(2,1,1,3 ; 1,2,1,1), B_{8}(2,1,1,3 ; 2,1,1,1), B_{8}(2,1,1,4 ; 1,1,1,1), B_{8}(2,1,2,1 ; 1,1,1,3)$.

(292) $B_{8}(2,1,2,1 ; 1,1,2,2), B_{8}(2,1,2,1 ; 1,1,3,1), B_{8}(2,1,2,1 ; 2,1,1,2), B_{8}(2,1,2,2 ; 1,1,2,1)$.

(293) $B_{8}(2,1,2,2 ; 2,1,1,1), B_{8}(2,1,2,3 ; 1,1,1,1), B_{8}(2,1,3,1 ; 1,1,1,2), B_{8}(2,1,3,1 ; 2,1,1,1)$.

(294) $B_{8}(2,1,4,1 ; 1,1,1,1), B_{8}(2,2,1,1 ; 1,1,3,1), B_{8}(2,2,1,1 ; 1,2,2,1), B_{8}(2,2,1,1 ; 1,3,1,1)$.

(295) $B_{8}(2,2,1,1 ; 2,2,1,1), B_{8}(2,2,1,2 ; 1,1,2,1), B_{8}(2,2,1,3 ; 1,1,1,1), B_{8}(2,2,2,1 ; 2,1,1,1)$.

(296) $B_{8}(2,2,2,2 ; 1,1,1,1), B_{8}(2,2,3,1 ; 1,1,1,1), B_{8}(2,3,1,1 ; 1,2,1,1), B_{8}(2,3,1,1 ; 2,1,1,1)$.

(297) $B_{8}(2,3,2,1 ; 1,1,1,1), B_{8}(2,4,1,1 ; 1,1,1,1), B_{8}(3,1,1,1 ; 1,1,2,2), B_{8}(3,1,1,1 ; 1,1,3,1)$.

(298) $B_{8}(3,1,1,1 ; 1,2,2,1), B_{8}(3,1,1,1 ; 2,1,2,1), B_{8}(3,1,1,1 ; 2,2,1,1), B_{8}(3,1,1,2 ; 1,1,1,2)$.

(299) $B_{8}(3,1,1,2 ; 1,1,2,1), B_{8}(3,1,1,2 ; 1,2,1,1), B_{8}(3,1,1,2 ; 2,1,1,1), B_{8}(3,1,1,3 ; 1,1,1,1)$.

(300) $B_{8}(3,1,2,1 ; 1,1,2,1), B_{8}(3,1,2,2 ; 1,1,1,1), B_{8}(3,2,1,1 ; 1,1,2,1), B_{8}(3,2,1,1 ; 2,1,1,1)$.

(301) $B_{8}(3,2,1,2 ; 1,1,1,1), B_{8}(3,2,2,1 ; 1,1,1,1), B_{8}(4,1,1,1 ; 1,1,1,2), B_{8}(4,1,1,1 ; 1,1,2,1)$.

(302) $B_{8}(4,1,1,1 ; 1,2,1,1), B_{8}(4,1,1,1 ; 2,1,1,1), B_{8}(4,1,1,2 ; 1,1,1,1), B_{8}(4,1,2,1 ; 1,1,1,1)$.

(303) $B_{8}(4,2,1,1 ; 1,1,1,1), B_{8}(5,1,1,1 ; 1,1,1,1)$.

(304) $B_{9}(1,1,1,1 ; 1,1,1,1 ; 1)$.

(305) $B_{9}(1,1,1,1 ; 1,1,1,1 ; 2), B_{9}(1,1,1,2 ; 1,1,1,1 ; 1), B_{9}(1,1,2,1 ; 1,1,1,1 ; 1)$.

(306) $B_{9}(1,2,1,1 ; 1,1,1,1 ; 1), B_{9}(2,1,1,1 ; 1,1,1,1 ; 1)$.

(307) $B_{9}(1,1,1,1 ; 1,1,1,1 ; 3), B_{9}(1,1,1,2 ; 1,1,1,1 ; 2), B_{9}(1,1,1,2 ; 1,1,1,2 ; 1)$.

(308) $B_{9}(1,1,1,3 ; 1,1,1,1 ; 1), B_{9}(1,1,2,1 ; 1,1,1,1 ; 2), B_{9}(1,1,2,1 ; 1,1,1,2 ; 1)$.

(309) $B_{9}(1,1,2,2 ; 1,1,1,1 ; 1), B_{9}(1,1,3,1 ; 1,1,1,1 ; 1), B_{9}(1,2,1,1 ; 1,1,1,1 ; 2)$.

(310) $B_{9}(1,2,1,1 ; 1,1,1,2 ; 1), B_{9}(1,2,1,1 ; 1,1,2,1 ; 1), B_{9}(1,2,1,1 ; 1,2,1,1 ; 1)$.

(311) $B_{9}(1,2,1,2 ; 1,1,1,1 ; 1), B_{9}(1,2,2,1 ; 1,1,1,1 ; 1), B_{9}(1,3,1,1 ; 1,1,1,1 ; 1)$.

(312) $B_{9}(2,1,1,1 ; 1,1,1,1 ; 2), B_{9}(2,1,1,1 ; 1,1,2,1 ; 1), B_{9}(2,1,1,1 ; 1,2,1,1 ; 1)$.

(313) $B_{9}(2,1,1,2 ; 1,1,1,1 ; 1), B_{9}(2,2,1,1 ; 1,1,1,1 ; 1), B_{9}(3,1,1,1 ; 1,1,1,1 ; 1)$.

(314) $B_{9}(1,1,1,1 ; 1,1,1,1 ; 4), B_{9}(1,1,1,2 ; 1,1,1,1 ; 3), B_{9}(1,1,1,2 ; 1,1,1,2 ; 2)$.

(315) $B_{9}(1,1,1,3 ; 1,1,1,2 ; 1), B_{9}(1,1,1,4 ; 1,1,1,1 ; 1), B_{9}(1,1,2,1 ; 1,1,1,1 ; 3)$.

(316) $B_{9}(1,1,2,1 ; 1,1,1,3 ; 1), B_{9}(1,1,2,2 ; 1,1,1,1 ; 2), B_{9}(1,1,2,2 ; 1,1,1,2 ; 1)$.

(317) $B_{9}(1,1,2,3 ; 1,1,1,1 ; 1), B_{9}(1,1,3,1 ; 1,1,1,2 ; 1), B_{9}(1,1,3,2 ; 1,1,1,1 ; 1)$.

(318) $B_{9}(1,1,4,1 ; 1,1,1,1 ; 1), B_{9}(1,2,1,1 ; 1,1,1,1 ; 3), B_{9}(1,2,1,1 ; 1,1,1,2 ; 2)$.

(319) $B_{9}(1,2,1,1 ; 1,1,2,1 ; 2), B_{9}(1,2,1,1 ; 1,2,1,1 ; 2), B_{9}(1,2,1,2 ; 1,1,1,1 ; 2)$.

(320) $B_{9}(1,2,1,2 ; 1,2,1,1 ; 1), B_{9}(1,2,2,1 ; 1,1,1,1 ; 2), B_{9}(1,2,2,1 ; 1,1,1,2 ; 1)$.

(321) $B_{9}(1,2,2,1 ; 1,2,1,1 ; 1), B_{9}(1,2,2,2 ; 1,1,1,1 ; 1), B_{9}(1,3,1,1 ; 1,1,1,1 ; 2)$.

(322) $B_{9}(1,3,1,1 ; 1,1,1,2 ; 1), B_{9}(1,3,1,1 ; 1,1,2,1 ; 1), B_{9}(1,3,1,1 ; 1,2,1,1 ; 1)$.

(323) $B_{9}(1,3,1,2 ; 1,1,1,1 ; 1), B_{9}(1,3,2,1 ; 1,1,1,1 ; 1), B_{9}(1,4,1,1 ; 1,1,1,1 ; 1)$.

(324) $B_{9}(2,1,1,1 ; 1,1,1,1 ; 3), B_{9}(2,1,1,1 ; 1,1,3,1 ; 1), B_{9}(2,1,1,1 ; 1,2,1,1 ; 2)$. 
(325) $B_{9}(2,1,1,1 ; 1,3,1,1 ; 1), B_{9}(2,1,1,2 ; 1,1,2,1 ; 1), B_{9}(2,1,1,2 ; 1,2,1,1 ; 1)$.

(326) $B_{9}(2,1,1,3 ; 1,1,1,1 ; 1), B_{9}(2,2,1,1 ; 1,1,1,1 ; 2), B_{9}(2,2,1,1 ; 1,1,2,1 ; 1)$.

(327) $B_{9}(2,2,1,1 ; 1,2,1,1 ; 1), B_{9}(2,2,1,2 ; 1,1,1,1 ; 1), B_{9}(2,3,1,1 ; 1,1,1,1 ; 1)$.

(328) $B_{9}(3,1,1,1 ; 1,1,2,1 ; 1), B_{9}(3,1,1,2 ; 1,1,1,1 ; 1), B_{9}(4,1,1,1 ; 1,1,1,1 ; 1)$.

(329) $B_{10}(1,1,1,1,1 ; 1,1,1,1,1)$.

(330) $B_{10}(1,1,1,1,2 ; 1,1,1,1,1), B_{10}(1,1,1,2,1 ; 1,1,1,1,1), B_{10}(1,1,2,1,1 ; 1,1,1,1,1)$.

(331) $B_{10}(1,2,1,1,1 ; 1,1,1,1,1), B_{10}(2,1,1,1,1 ; 1,1,1,1,1)$.

(332) $B_{10}(1,1,1,1,3 ; 1,1,1,1,1), B_{10}(1,1,1,2,1 ; 1,1,1,1,2), B_{10}(1,1,1,2,1 ; 1,1,1,2,1)$.

(333) $B_{10}(1,1,1,2,2 ; 1,1,1,1,1), B_{10}(1,1,1,3,1 ; 1,1,1,1,1), B_{10}(1,1,2,1,1 ; 1,1,1,1,2)$.

(334) $B_{10}(1,1,2,2,1 ; 1,1,1,1,1), B_{10}(1,1,3,1,1 ; 1,1,1,1,1), B_{10}(1,2,1,1,1 ; 1,1,2,1,1)$.

(335) $B_{10}(1,2,1,1,1 ; 1,2,1,1,1), B_{10}(1,2,1,1,2 ; 1,1,1,1,1), B_{10}(1,2,2,1,1 ; 1,1,1,1,1)$.

(336) $B_{10}(1,3,1,1,1 ; 1,1,1,1,1), B_{10}(2,1,1,1,1 ; 1,1,1,2,1), B_{10}(2,1,1,1,1 ; 1,1,2,1,1)$.

(337) $B_{10}(2,1,1,1,1 ; 2,1,1,1,1), B_{10}(2,1,1,1,2 ; 1,1,1,1,1), B_{10}(2,1,1,2,1 ; 1,1,1,1,1)$.

(338) $B_{10}(2,2,1,1,1 ; 1,1,1,1,1), B_{10}(3,1,1,1,1 ; 1,1,1,1,1)$.

(339) $B_{11}(1,1,1,1,1 ; 1,1,1,1,1 ; 1)$.

(340) $B_{11}(1,1,1,1,1 ; 1,1,1,1,1 ; 2), B_{11}(1,1,1,1,2 ; 1,1,1,1,1 ; 1)$.

(341) $B_{11}(1,1,2,1,1 ; 1,1,1,1,1 ; 1), B_{11}(1,2,1,1,1 ; 1,1,1,1,1 ; 1)$.

(342) $B_{12}(1,1,1,1,1,1 ; 1,1,1,1,1,1)$.

Proof. One can check that for every 1575 above graphs $G, \lambda_{1}(G)>0, \lambda_{2}(G)>0$ and $\lambda_{3}(G)<0$. Now assume that $G$ is a connected graph of order $n$ such that $\lambda_{1}(G)>0, \lambda_{2}(G)>0$ and $\lambda_{3}(G)<0$. Since

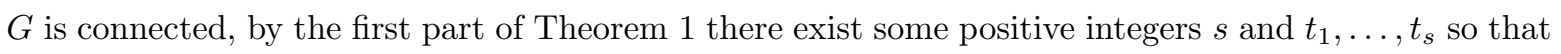
$3 \leq s \leq 12$ and $t_{1}+\cdots+t_{s}=n$ and $G \cong G_{s}\left[K_{t_{1}}, \ldots, K_{t_{s}}\right]$. Now we obtain all positive integers $s$ and $t_{1}, \ldots, t_{s}$ where $3 \leq s \leq 12$ and $t_{1}+\cdots+t_{s} \leq 12$ such that

$$
\lambda_{1}\left(G_{s}\left[K_{t_{1}}, \ldots, K_{t_{s}}\right]\right)>0, \quad \lambda_{2}\left(G_{s}\left[K_{t_{1}}, \ldots, K_{t_{s}}\right]\right)>0, \text { and } \lambda_{3}\left(G_{s}\left[K_{t_{1}}, \ldots, K_{t_{s}}\right]\right)<0 .
$$

We investigate the case $s=5$, the other cases similarly is investigated. In other words assume that

$$
\lambda_{1}\left(G_{5}\left[K_{t_{1}}, \ldots, K_{t_{5}}\right]\right)>0, \quad \lambda_{2}\left(G_{5}\left[K_{t_{1}}, \ldots, K_{t_{5}}\right]\right)>0 \text {, and } \lambda_{3}\left(G_{5}\left[K_{t_{1}}, \ldots, K_{t_{5}}\right]\right)<0 .
$$

By the notation of Definition [3, since $B_{5}\left(t_{1}, t_{2} ; t_{3}, t_{4} ; t_{5}\right) \cong G_{5}\left[K_{t_{1}}, \ldots, K_{t_{5}}\right]$, it suffices to obtain all values of $t_{1}, \ldots, t_{5}$ such that

$$
\lambda_{1}\left(B_{5}\left(t_{1}, t_{2} ; t_{3}, t_{4} ; t_{5}\right)\right)>0, \lambda_{2}\left(B_{5}\left(t_{1}, t_{2} ; t_{3}, t_{4} ; t_{5}\right)\right)>0 \text {, and } \lambda_{3}\left(B_{5}\left(t_{1}, t_{2} ; t_{3}, t_{4} ; t_{5}\right)\right)<0 \text {. }
$$

By Theorem $\square$ we obtain that

$$
P\left(B_{5}\left(t_{1}, t_{2} ; t_{3}, t_{4} ; t_{5}\right), \lambda\right)=(\lambda+1)^{t_{1}+\cdots+t_{5}} \Phi\left(t_{1}, \ldots, t_{5}, \lambda\right),
$$




\begin{tabular}{|l|c|c|c|c|c|c|}
\hline The number of $\cdots$ graphs of order $n$ & $n=1$ & $n=2$ & $n=3$ & $n=4$ & $n=5$ & $n=6$ \\
\hline connected & 1 & 1 & 2 & 6 & 21 & 112 \\
\hline connected and integral & 1 & 1 & 1 & 2 & 3 & 6 \\
\hline connected with $\lambda_{2}>0$ and $\lambda_{3}<0$ & 0 & 0 & 0 & 2 & 6 & 15 \\
\hline connected integral with $\lambda_{2}>0$ and $\lambda_{3}<0$ & 0 & 0 & 0 & 0 & 0 & 0 \\
\hline$B_{3}(. ; . ;$.$) graphs with \lambda_{2}>0$ and $\lambda_{3}<0$ & 0 & 0 & 0 & 1 & 3 & 5 \\
\hline$B_{4}(. ;$.$) graphs with \lambda_{2}>0$ and $\lambda_{3}<0$ & 0 & 0 & 0 & 1 & 2 & 6 \\
\hline$B_{5}(. ; . ;$.$) graphs with \lambda_{2}>0$ and $\lambda_{3}<0$ & 0 & 0 & 0 & 0 & 1 & 3 \\
\hline$B_{6}(. ;$.$) graphs with \lambda_{2}>0$ and $\lambda_{3}<0$ & 0 & 0 & 0 & 0 & 0 & 1 \\
\hline$B_{7}(. ; . ;$.$) graphs with \lambda_{2}>0$ and $\lambda_{3}<0$ & 0 & 0 & 0 & 0 & 0 & 0 \\
\hline$B_{8}(. ;$.$) graphs with \lambda_{2}>0$ and $\lambda_{3}<0$ & 0 & 0 & 0 & 0 & 0 & 0 \\
\hline$B_{9}(. ; . ;$.$) graphs with \lambda_{2}>0$ and $\lambda_{3}<0$ & 0 & 0 & 0 & 0 & 0 & 0 \\
\hline$B_{10}(. ;$.$) graphs with \lambda_{2}>0$ and $\lambda_{3}<0$ & 0 & 0 & 0 & 0 & 0 & 0 \\
\hline$B_{11}(. ; . ;$.$) graphs with \lambda_{2}>0$ and $\lambda_{3}<0$ & 0 & 0 & 0 & 0 & 0 & 0 \\
\hline$B_{12}(. ;$.$) graphs with \lambda_{2}>0$ and $\lambda_{3}<0$ & 0 & 0 & 0 & 0 & 0 & 0 \\
\hline \hline
\end{tabular}

TABLE 1. The table of unlabeled connected graphs with some properties

where $\Phi\left(t_{1}, \ldots, t_{5}, \lambda\right)$ is the characteristic polynomial of the matrix $M\left(t_{1}, \ldots, t_{5}\right)$,

$$
M\left(t_{1}, \ldots, t_{5}\right)=\left(\begin{array}{ccccc}
t_{1}-1 & t_{2} & 0 & 0 & t_{5} \\
t_{1} & t_{2}-1 & 0 & t_{4} & t_{5} \\
0 & 0 & t_{3}-1 & t_{4} & t_{5} \\
0 & t_{2} & t_{3} & t_{4}-1 & t_{5} \\
t_{1} & t_{2} & t_{3} & t_{4} & t_{5}-1
\end{array}\right) .
$$

Using Equation (3) we conclude that the Equation (피) holds if and only if

$$
\lambda_{1}\left(M\left(t_{1}, \ldots, t_{5}\right)\right)>0, \quad \lambda_{2}\left(M\left(t_{1}, \ldots, t_{5}\right)\right)>0, \text { and } \lambda_{3}\left(M\left(t_{1}, \ldots, t_{5}\right)\right)<0 .
$$

By Maple one can obtain all positive integers $t_{1}, \ldots, t_{5}$ such that $t_{1}+\cdots+t_{5} \leq 12$ and the Equation (田) holds. This completes the proof.

Remark 3.2. We note that among 1575 connected graphs of Theorem $\mathbf{3}$ just two of them are integral. These graphs are $B_{3}(2 ; 2 ; 3)$ and $B_{3}(3 ; 3,2)$. In fact

$\operatorname{Spec}\left(B_{3}(2 ; 2 ; 3)\right)=\{5,1,-1,-1,-1,-1,-2\}$, and $\operatorname{Spec}\left(B_{3}(3 ; 3 ; 2)\right)=\{5,2,-1,-1,-1,-1,-1,-2\}$.

We end the paper by comparing the number of connected graphs, the number of integral connected graphs and the number of connected graphs with positive second largest eigenvalue and negative third largest eigenvalue, see Tables $\mathrm{U}$ and $\boldsymbol{\nabla}$. For every integer $n \geq 1$, let $a_{n}$ be the number of connected 


\begin{tabular}{|l|c|c|c|c|c|c|}
\hline The number of $\cdots$ graphs of order $n$ & $n=7$ & $n=8$ & $n=9$ & $n=10$ & $n=11$ & $n=12$ \\
\hline connected & 853 & 11117 & 261080 & $>10^{7}$ & $>10^{9}$ & $>10^{11}$ \\
\hline connected and integral & 7 & 22 & 24 & 83 & 113 & $?$ \\
\hline connected with $\lambda_{2}>0$ and $\lambda_{3}<0$ & 31 & 66 & 129 & 255 & 444 & 627 \\
\hline connected integral with $\lambda_{2}>0$ and $\lambda_{3}<0$ & 1 & 1 & 0 & 0 & 0 & 0 \\
\hline$B_{3}(. ; . ;$.$) graphs with \lambda_{2}>0$ and $\lambda_{3}<0$ & 8 & 11 & 15 & 19 & 24 & 29 \\
\hline$B_{4}(. ;$.$) graphs with \lambda_{2}>0$ and $\lambda_{3}<0$ & 10 & 19 & 28 & 43 & 56 & 68 \\
\hline$B_{5}(. ; . ;$.$) graphs with \lambda_{2}>0$ and $\lambda_{3}<0$ & 9 & 19 & 37 & 61 & 87 & 101 \\
\hline$B_{6}(. ;$.$) graphs with \lambda_{2}>0$ and $\lambda_{3}<0$ & 3 & 12 & 28 & 64 & 109 & 142 \\
\hline$B_{7}(. ; . ;$.$) graphs with \lambda_{2}>0$ and $\lambda_{3}<0$ & 1 & 4 & 16 & 42 & 87 & 119 \\
\hline$B_{8}(. ;$.$) graphs with \lambda_{2}>0$ and $\lambda_{3}<0$ & 0 & 1 & 4 & 20 & 54 & 98 \\
\hline$B_{9}(. ; . ;$.$) graphs with \lambda_{2}>0$ and $\lambda_{3}<0$ & 0 & 0 & 1 & 5 & 21 & 45 \\
\hline$B_{10}(. ;$.$) graphs with \lambda_{2}>0$ and $\lambda_{3}<0$ & 0 & 0 & 0 & 1 & 5 & 20 \\
\hline$B_{11}(. ; . ;$.$) graphs with \lambda_{2}>0$ and $\lambda_{3}<0$ & 0 & 0 & 0 & 0 & 1 & 4 \\
\hline$B_{12}(. ;$.$) graphs with \lambda_{2}>0$ and $\lambda_{3}<0$ & 0 & 0 & 0 & 0 & 0 & 1 \\
\hline \hline
\end{tabular}

TABLE 2. The table of unlabeled connected graphs with some properties

integral graphs of order $n$ and $b_{n}$ be the number of connected graphs of order $n$ with $\lambda_{2}>0$ and $\lambda_{3}<0$. According to the third and the fourth row of the Tables $\mathbb{U}$ and $\nabla$ we conclude that for every $4 \leq n \leq 11$, $a_{n} \leq b_{n}$. Thus we pose the following problem:

Problem 1. Is it true that for every $n \geq 3, a_{n} \leq b_{n}$ ?

\section{Acknowledgements}

This paper is related to a research project that supported by Islamic Azad University Marvdasht Branch. The research of the second author was in part supported by a grant (No. 96050011) from School of Mathematics, Institute for Research in Fundamental Sciences (IPM).

\section{REFERENCES}

[1] M.R. Oboudi, Bipartite graphs with at most six non-zero eigenvalues, Ars Mathematica Contemporanea 11 (2016) $315-325$.

[2] M.R. Oboudi, Characterization of graphs with exactly two non-negative eigenvalues, Ars Mathematica Contemporanea 12 (2017) 271-286.

[3] M.R. Oboudi, On the third largest eigenvalue of graphs, Linear Algebra and its Applications 503 (2016) 164-179

[4] M. Petrović, Graphs with a small number of nonnegative eienvalues, Graphs and Combinatorics 15 (1999) $221-232$.

[5] J. H. Smith, Symmetry and multiple eigenvalues of graphs, Glas. Mat., Ser. III 12 (1977) No. 1, 3-8. 


\section{Tajedin Derikvand}

Department of Mathematics, Marvdasht Branch, Islamic Azad University, Marvdasht, Iran

Email: derikvand@miau.ac.ir

\section{Mohammad Reza Oboudi}

Department of Mathematics, College of Sciences, Shiraz University, Shiraz, 71457-44776, Iran

and School of Mathematics, Institute for Research in Fundamental Sciences (IPM), P.O. Box 19395-5746, Tehran, Iran

Email: mr_oboudi@yahoo.com, mr_oboudi@shirazu.ac.ir 